\title{
Mathematical Modeling of the Proliferation Gradient in MultiCellular Tumor Spheroids
}

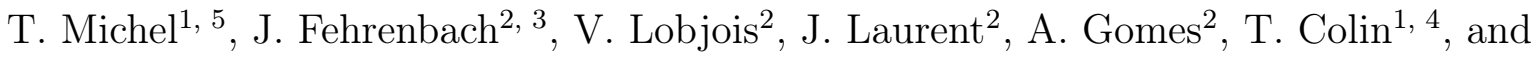 \\ C. Poignard ${ }^{* 1}$ \\ ${ }^{1}$ Univ. Bordeaux, IMB, UMR 5251, F-33400, Talence, France, INRIA Bordeaux-Sud-Ouest, F-33400, \\ Talence, France \\ ${ }^{2}$ ITAV-USR3505, Université de Toulouse, CNRS, UPS, Toulouse, France \\ ${ }^{3}$ Institut de Mathématiques de Toulouse, Université de Toulouse, CNRS, UPS, Toulouse, France \\ ${ }^{4}$ Bordeaux INP, IMB, UMR 5251, F-33400, Talence, France \\ ${ }^{5}$ The Center for Mathematical Modeling and Data Science, Osaka University, Toyonaka, Japan
}

\begin{abstract}
MultiCellular Tumor Spheroids are 3D cell cultures that can accurately reproduce the behavior of solid tumors. It has been experimentally observed that large spheroids exhibit a decreasing gradient of proliferation from the periphery to the center of these multicellular 3D models: the proportion of proliferating cells is higher in the periphery while the nonproliferating quiescent cells increase in depth. In this paper, we propose to investigate the key mechanisms involved in the establishment of this gradient with a Partial Differential Equations model that mimics the experimental set-up of growing spheroids under different nutrients supply conditions. The model consists of mass balance equations on the two cell populations observed in the data: the proliferating cells and the quiescent cells. The spherical symmetry is used to rewrite the model in radial and relative coordinates. Thanks to a rigorous data postprocessing the model is then fit and compared quantitatively with the experimental quantification of the percentage of proliferating cells from EdU immunodetection on 2D spheroid cryosection images. The results of this calibration show that the proliferation gradient observed in spheroids can be quantitatively reproduced by our model.
\end{abstract}

Keywords: Tumor growth model, nonlinear advection-reaction equations, proliferation gradient in tumors

\section{Introduction}

For the last decades, myriad of cytotoxic drugs, targeted therapies, and immunotherapies have been developed to control tumors expansion with promising results. Generally speaking, these cytotoxic drugs have been mainly designed in vitro to target proliferating cells of a specific disease. It is commonly accepted that tumor heterogeneity may favor the emergence of resistant phenotypes, leading to treatment failures. The mechanisms of the emergence of these phenotypes are still unclear and in all probability multifactorial. One can cite for instance the heterogeneous drug distribution within the tumor due to the interstitial fluid pressure [4, 39]. Moreover, the different phases (proliferating or quiescent phases) of the cells composing the tumors lead to different cell response to drugs within the tumor. In particular, it is worth noting that most of

\footnotetext{
${ }^{*}$ Corresponding author: clair.poignard@inria.fr
} 
the therapies target the highly proliferating cells by inducing DNA damages and consequently cell death thanks to the DNA damage response and cell cycle regulation. However, large tumors exhibit quiescent cells that have stopped proliferating due to the lack of nutrients, oxygen, and growth factor in depth within tumors. Such quiescent cells are less affected by drugs (and even radiotherapy), which can promote the emergence of resistant phenotypes.

There is currently an increasing interest in using computational modeling on tumor growth and response to therapy. In this context, it appears essential to consider the evolution of the tumor cell heterogeneity in terms of proliferation. For that purpose, MultiCellular Tumor Spheroids (MCTS) cell cultures are used to understand the dynamics and the phenomena involved in tumor heterogeneity and drug penetration. The 3D structure of MCTS makes them an interesting mesoscale biological model between 2D monolayer cell cultures and animals models [37. Interestingly the gene expression observed in MCTS is closer to patients profile gene expression than the profiles observed in 2D monolayer cultures [27, 35]. In addition, in spheroids with a diameter larger than a few hundreds of microns the lacks of nutrient, oxygen, and growth factors in the center of the spheroid induce the establishment of a proliferation gradient with an increasing proportion of quiescent cells in depth [21, 46]. Unlike 2D cell cultures, the MCTS spatial heterogeneity mimics what is observed in tumors [20]. It has also been mentioned that MCTS response to a drug is more predictive of the drug efficiency than the response observed in $2 \mathrm{D}$ cell cultures 32 . For these reasons, MCTS are well fitted to understand the mechanisms in the early stage of micro-tumors and to evaluate new drugs [28].

It is now well established that well-designed mathematical modeling can be useful to test and possibly explain new biological hypotheses. Regarding the tumor spheroids, mathematical modeling can provide quantitative information to understand the properties of tumor spheroids. For instance, the use of classical Ordinary Differential Equations (ODEs) models such as the Gompertz model, the power law model or the logistic model provide good descriptions of the tumor volume evolution [5]. ODE models are also used to describe the heterogeneity within a tumor by using several compartment of cells, such as proliferating cells and quiescent cells [48]. However, these models are not designed for describing the proliferation gradient that is observed in tumor spheroids. More complex models are needed to account for such a gradient [11, 26, 29, 36] as well as the drug penetration in 3D tumors [16].

Mathematical models can be classified into two main approaches: the discrete models and the continuous models. Reviews and comparisons between these approaches can be found in the literature [9, 22, 42, 44]. Discrete models such as cellular automata [17] or agent-based models [18, 19, 43] are well-fitted to reproduce the evolution of spheroids, as they describe cells individually. However the number of parameters needed in these models and their computational cost make them hardly extendable to the in vivo scale. In continuous models, the spheroid is described as a continuum of cells by using Partial Differential Equations (PDEs) on cell densities and nutrient concentration. Some of the PDE models, which are based on reaction-diffusion equation, are used for modeling cell motility [8, 23, 47, whereas other PDE models, which are based on mass balance equations, are used for modeling proliferation induced growth of the spheroid [12, 38, 45, 50. Hybrid models combine discrete and continuous approaches [1, 3, 31, 40]. They can account for numerous biological phenomenon, but their are very sensitive to model parameters, making them hardly fittable with quantitative biological data in simple experimental set-up.

In some recent studies, experimental data and mathematical models are combined in order to understand the dynamics of spheroid growth. In [36, Loessner et al. focused on the growth of the spheroids submitted to cytotoxic drugs but they did not account for the spatial heterogeneity observed within a spheroid. In [26], Grimes et al. proposed a model describing the position of the viable rim and they estimated the rates of oxygen consumption from spheroids. The authors 
modeled the spatial heterogeneity as a binary process, consisting of a hypoxic region surrounded by a proliferating region without accounting for the gradient of proliferation, as observed in the experiments [33, 24]. Jagiella et al. developed an agent-based model to describe quantitatively the gradient of proliferation by incorporating numerous phenomena [29]. However, the model complexity and the number of parameters of their model make it very sensitive to parameters variability and thus difficult to calibrate with other experimental configurations and even worse with in vivo studies.

The goal of this paper is twofold. On the one hand, we use a continuous PDE model of spheroid growth accounting for the influence of the nutrient supply. This model makes appear the proliferation gradient as experimentally observed in tumor spheroids [33]. It is built in the same vein as the model of Colin, Saut, et al. (see for instance [6, 41, 34]). It consists of mass balance equations on several populations of cells, which are in our case the proliferating cells and quiescent cells. In general, this kind of models requires the choice of a proper closure equation for the velocity field of the mass balance equations. For instance, Darcy's law can be considered [2, 25] or other closures as for viscoelastic fluid can be used [6, 15]. It is worth noting that cells that are considered not mechanosensitive.

Interestingly, in the framework of radial symmetry, the velocity field is entirely determined by its divergence, therefore no assumption on the rheology of the tumor spheroid is needed. Even though our model is similar to classical models such as the models proposed by the research groups of Ambrosi, Byrne, Preziosi (see for instance [2, 10, 12, 14, 49]), we propose to rewrite the model by using the relative position inside the spheroid, instead of the absolute radial position. The use of these coordinates makes it easier to distinguish between the global effect of cell proliferation on tumor growth and the local effect of the proliferation gradient on the local cell velocity.

On the other hand, we propose a rigorous methodology to use the experimental data [33]. These data consist of the measurements of spheroid growth kinetics and in the immunodetection on spheroids cryosections of proliferating cells after EdU incorporation. The interesting features of this staining is to provide the spatial distribution of the proliferating and quiescent cells (nonpositive for the EdU marker) within a spheroid. The method used to extract data from the experimental images and the accuracy of this method are provided in 33 . The discrete nature of the data makes the comparison with a continuous model not straightforward. We propose a method consisting of computing the fraction of proliferating cells between two relative radial positions in the spheroid and comparing these fractions with the corresponding integrals of the proliferating cell density obtained by the simulation of the model. In addition, we propose an algorithm to fit the model with the data.

The outline of the present paper is as follows. In Section 2, we present the mathematical model used in this study and we rewrite the PDEs in the relative radial coordinates to benefit from the radial symmetry of spheroids. Then Section 3 presents the methodology to link the experimental data to the model and we give the calibration algorithm. We apply this method to fit the model with the data in Section 4 , showing the consistency of our approach. We conclude the paper with a discussion section.

\section{The Mathematical model}

In this section, we present the mathematical model that has been developed for the purpose of modeling the experimental data on tumor spheroids. In the first paragraph, we present the main assumptions of the model and their mathematical writing. We rewrite these equations in relative radial coordinates in the second paragraph and we perform non-dimensionalization of the equations in the third paragraph. Then, in the last paragraph, we present the simplifications 
of the model that are used for the calibration of the model in Section 3 .

\subsection{Model description}

Denote the tumor spheroid at time $t$ by $\Omega(t)$ and assume that $\Omega(t)$ is a sphere of radius $R(t)$. In this paper, we are working on tumor spheroids which are composed of two kinds of tumor cells. We make the assumptions that the spheroid consists of a continuum of live cells [2, 10, 12, 14, 49]. The cells are assumed to have same size and mass and are assumed to be incompressible, i.e their density, defined as the number of cells per volume unit, is constant in space and time.

For any time $t$ and any position $x$, we denote by

- $P(t, x)$ the density (in $[0,1]$, dimensionless) of proliferating cells, the cells which are positive for EdU marker,

- $Q(t, x)$, the density (in $[0,1]$, dimensionless) of quiescent cells, the cells which are negative to EdU marker (these cells did not divide at least during the past 24 hours).

The proliferating cells are assumed to proliferate at a rate $\gamma_{0}(C(t, x))$, which depends on the local nutrient, oxygen, and growth factor concentration $C(t, x)$. Under hypoxia condition, when this local concentration $C$ is low, some cells stop their proliferation and become quiescent. We denote by $\gamma_{1}(C(t, x))$ the switch rate at which proliferating cells enter the quiescent state. The functions $\gamma_{0}$ and $\gamma_{1}$ are assumed to be $C^{\infty}$ and non-negative functions of $C$. $\gamma_{0}$ is assumed to be increasing while $\gamma_{1}$ is assumed to be decreasing.

The growth of the spheroid is modeled by assuming that cell proliferation induces a passive movement of the cells [14. We denote by $\mathbf{v}(t, x)$ the passive velocity of the cells. Under these assumptions, the tumor cell densities satisfy the following mass balance equations in $\Omega(t)$ :

$$
\begin{aligned}
& \partial_{t} P+\nabla \cdot(\mathbf{v} P)=\gamma_{0}(C) P-\gamma_{1}(C) P \\
& \partial_{t} Q+\nabla \cdot(\mathbf{v} Q)=\gamma_{1}(C) P .
\end{aligned}
$$

We also assume that the tumor spheroid is just composed of these two kinds of cells. This assumption leads to the following saturation equation

$$
P(t, x)+Q(t, x)=1, \quad \text { in } \Omega(t) .
$$

By summing the equations on cells densities (1) and (2), and by using the previous saturation assumption we obtain the following equation for the divergence of the velocity field:

$$
\nabla \cdot \mathbf{v}=\gamma_{0}(C) P .
$$

The concentration of nutrients, oxygen, and growth factor in the spheroid is denoted by $C$. This concentration is supposed to satisfy a stationary diffusion equation. This choice is relevant because the diffusion of nutrient, oxygen, and growth factor in the spheroid is very fast compared to the time scale of proliferation, which is about one day [11, 12]. We assume that proliferating cells consume nutrient, oxygen, and growth factor at a constant rate denoted by $\alpha$. The equation satisfied by $C$ takes the following form:

$$
-\Delta C=-\alpha P C
$$

We assume that the external concentration of nutrients, oxygen, and growth factor is constant, equal to $C_{0}$. 
Remark 2.1. Cell proliferation is a complex multifactorial phenomenon. In our model, the variable $C$ represents the combined effect of the concentration of several proliferation promoters, such as nutrients, oxygen, and growth factor. The relative effect of these factors is not known. Therefore the variable $C$ and the external concentration $C_{0}$ cannot be explicitly expressed in terms of concentration of these quantities.

Remark 2.2 (Modeling assumptions). This modeling study is based upon the experiments of Laurent et al. [33]. The external concentration of nutrients has been experimentally set to avoid the cell necrosis. Therefore, we do not include a necrotic cell density. Moreover, since the external concentration has been set to a constant and no external support is assumed, this concentration is expected to decrease over time inside the spheroid. Therefore, the quiescent cells cannot resume to a proliferation state in the experiments, and this possibility was neither included in the model.

\subsection{Reformulation of the model in relative radial coordinates}

Under radial symmetry assumption, we rewrite the model in relative radial coordinates. For $t \geq 0$ and $x \in \Omega(t)$, we denote by

$$
r(t, x)=\frac{|x|}{R(t)} \in[0,1]
$$

the relative radial coordinate. For brevity, we still denote by $P$ and $Q$ the cell densities, by $\mathbf{v}$ the velocity, and by $C$ the concentration written in these coordinates.

Proposition 2.3 (The model in relative radial coordinates). By using the relative radial coordinates, we rewrite the model (1) - (5) as follows:

$$
\begin{aligned}
\Gamma_{P}(t, r) & =\int_{0}^{r} \gamma_{0}(C(t, \tilde{r})) P(t, \tilde{r}) \tilde{r}^{2} \mathrm{~d} \tilde{r}, \\
R(t) & =R^{0} \exp \left(\int_{t^{0}}^{t} \Gamma_{P}(\tilde{t}, 1) \mathrm{d} \tilde{t}\right), \\
\partial_{t} P+v \partial_{r} P & =\gamma_{0}(C) P(1-P)-\gamma_{1}(C) P, \\
v(t, r) & =\frac{1}{r^{2}}\left(\Gamma_{P}(t, r)-r^{3} \Gamma_{P}(t, 1)\right), \\
-\Delta_{r} C & =-\alpha R^{2} P C,
\end{aligned}
$$

where $\Delta_{r} C=\frac{1}{r^{2}} \partial_{r}\left(r^{2} \partial_{r} C\right)$ denotes the $3 D$ radial Laplacian of $C$. We consider the initial conditions $R\left(t^{0}\right)=R^{0}, P\left(t^{0}, \cdot\right)=P^{0}(\cdot)$ at initial time $t^{0}$ and the boundary conditions $\left.\partial_{r} C\right|_{r=0}=0$, $\left.C\right|_{r=1}=C_{0}$.

The method used to perform the numerical simulations of the model is based upon the standard Finite Difference method. For the sake of conciseness, it is detailed in Appendix 8.2,

Proof. To prove the equation satisfied by the radius $(6 \mathrm{~b})$, we integrate the saturation equation (3) over $\Omega(t)$, we differentiate in time and we use the equation satisfied by the cell densities (1) and (2). This leads to the following differential equation for the radius $R(t)$ :

$$
R^{\prime}(t)=R(t) \int_{0}^{1} \gamma_{0}(C(t, r)) P(t, r) r^{2} \mathrm{~d} r
$$

whose solution is given by (6b). 
To obtain the equation satisfied by the proliferating cell density 6c), we start by using equation (4) in (1) to reformulate the term $(\nabla \cdot \mathbf{v}) P$. We denote by $v_{r}$ the radial component of the velocity $\mathbf{v}$. By rewriting the equation (1) in the relative radial coordinate, we obtain equation (6c), where the relative velocity $v$ is given by

$$
v(t, r)=\frac{v_{r}(t, r)-r R^{\prime}(t)}{R(t)}
$$

This velocity corresponds to the local velocity relatively to the spheroid expansion velocity $R^{\prime}(t)$.

The formula (6d) for the above velocity $v$ is obtained by integration of equation (4) over the radial coordinate $r$ (radial symmetry assumption implies $v_{r}=0$ for $r=0$ ). This leads to a formula for the radial velocity $v_{r}$. Then using the above formula (7) in the definition of the velocity $v$ and the definition of $\Gamma_{P}$ given by (6a) leads to (6d).

The equation satisfied by the concentration (6e) is directly obtained from (5) written in radial coordinates, the radial symmetry assumption implies the Neumann boundary condition $\partial_{r} C(t, 0)=0$.

In the general case (e.g. asymmetric growth), one needs to add a closure equation for the velocity. Moreover, when the cells are assumed to be mechanosensitive, one needs to add an equation for the pressure. For instance, Darcy's law can be considered [2, 25]. Other closures have also been investigated [6, 15]. However, in our case we assume a radial symmetry and cells to be not mechanosensitive. Under these assumptions the equation satisfied by the velocity $v$ can be explicitly solved by using $(6 \mathrm{~d})$. Formula $(6 \mathrm{~d})$ is also useful to obtain a bound on the velocity $v$ (see Proposition 8.7 in Appendix 8.1). This bound is used in the numerical simulations of the model.

Proposition 2.4 (Estimate for the concentration). Denote by $P_{\min }(t)=\min _{r \in[0,1]} P(t, r)$. Thanks to the maximum principle satisfied by $C$ (see Appendix 8.1 Proposition 8.3), the following estimates hold:

$$
C(t, r) \leq C_{P_{\min }(t)}(t, r)=\frac{C_{0}}{r} \frac{\sinh \left(r \sqrt{\alpha P_{\min }(t) R(t)^{2}}\right)}{\sinh \left(\sqrt{\alpha P_{\min }(t) R(t)^{2}}\right)} .
$$

Thanks to the above proposition, when the value of $\sqrt{\alpha P_{\min }(t) R(t)^{2}}$ is large enough, the concentration is almost zero in a neighborhood of $r=0$. For instance, $C \simeq 0$ for $r \leq 0.4$ when $\sqrt{\alpha P_{\min }(t) R(t)^{2}}=10$ (see Figure 1). This qualitative behavior is similar to the one used in [26] but in our case we do not explicitly set the region of the zero concentration.

Remark 2.5 (Growth saturation). Under some conditions on the parameters of the model, growth saturation can be achieved. The general condition is that the integral

$$
\int_{t^{0}}^{t} \int_{0}^{1} \gamma_{0}(C(\tilde{t}, \tilde{r})) P(\tilde{t}, \tilde{r}) \tilde{r}^{2} \mathrm{~d} \tilde{r} \mathrm{~d} \tilde{t}
$$

is bounded. Since $\gamma_{0}$ is bounded, it is sufficient for instance that

$$
\int_{0}^{1} P(\tilde{t}, \tilde{r}) \tilde{r}^{2} \mathrm{~d} \tilde{r}=O\left(\frac{1}{\tilde{t}^{1+\varepsilon}}\right), \quad \text { with } \varepsilon>0
$$

If $\gamma_{0}\left(C_{0}\right)<\gamma_{1}\left(C_{0}\right)$, one can prove that such condition occurs and one can provide an upper bound for the radius (see Appendix 8.1 Proposition 8.6). In [12], the author studied the existence of a stationary solution for a similar model, accounting for proliferating and quiescent cells only. 


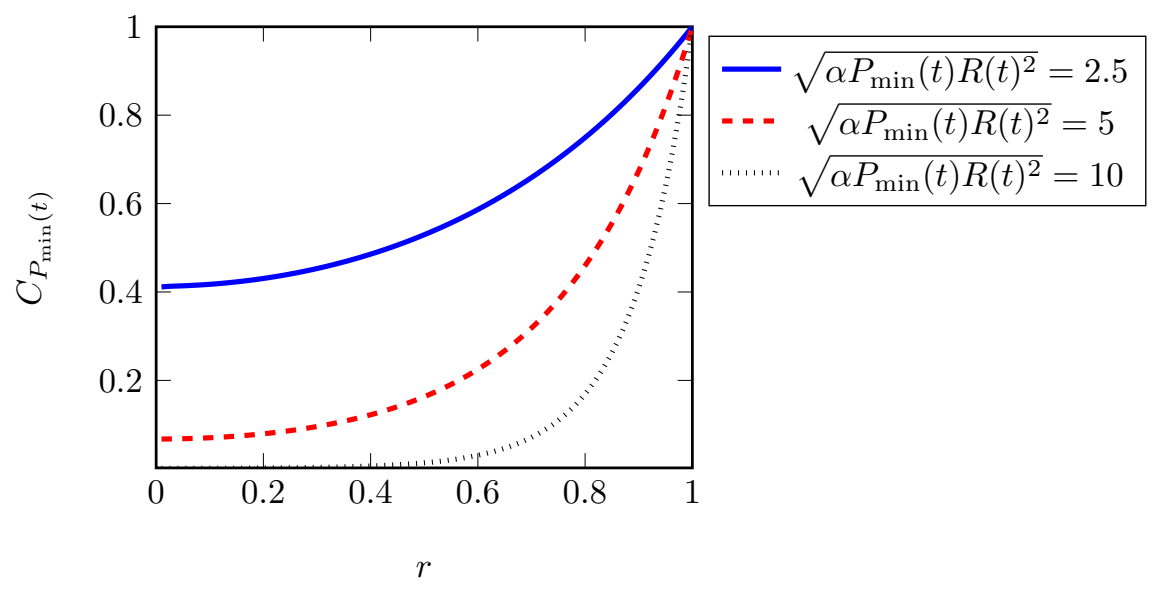

Figure 1: Plot of the upper bound for the concentration given by $C_{P_{\min }(t)}$ in Proposition 2.4

\subsection{Data driven non-dimensionalization}

We rescale the model equations by using the following quantities

$$
\hat{t}^{0}=t^{0} / t_{C}, \quad \hat{t}=t / t_{C}, \quad \hat{R}(\hat{t})=R(t) / R_{C}, \quad \hat{R}^{0}=R^{0} / R_{C},
$$

where $t_{C}=1$ day is a characteristic time (the cell division is of order 1 day in the experiments) and $R_{C}=100 \mu \mathrm{m}$ is a characteristic length (the radius of spheroids are of order $100 \mu \mathrm{m}$ ). We also consider the following quantities

$$
\begin{aligned}
\hat{\gamma}_{0}(\hat{C}) & =t_{C} \gamma_{0}(C), & \hat{\gamma}_{1}(\hat{C})=t_{C} \gamma_{1}(C), & \hat{\Gamma}_{P}(\hat{t}, r)=t_{C} \Gamma_{P}(t, r), \\
\hat{v}(\hat{t}, r)=t_{C} v(t, r), & \hat{C}(\hat{t}, r)=C(t, r) / C_{0}, & \hat{\alpha} & =\alpha R_{C}^{2} .
\end{aligned}
$$

These rescalings lead to the following system of equations

$$
\begin{aligned}
\hat{\Gamma}_{P}(\hat{t}, r) & =\int_{0}^{r} \hat{\gamma}_{0}(\hat{C}(\hat{t}, \tilde{r})) P(t, \tilde{r}) \tilde{r}^{2} \mathrm{~d} \tilde{r}, \\
\hat{R}(\hat{t}) & =\hat{R}^{0} \exp \left(\int_{\hat{t}^{0}}^{\hat{t}} \hat{\Gamma}_{P}(\tilde{t}, 1) \mathrm{d} \tilde{t}\right), \\
\partial_{\hat{t}} P+\hat{v} \partial_{r} P & =\hat{\gamma}_{0}(\hat{C}) P(1-P)-\hat{\gamma}_{1}(\hat{C}) P, \\
\hat{v}(\hat{t}, r) & =\frac{1}{r^{2}}\left(\hat{\Gamma}_{P}(\hat{t}, r)-r^{3} \hat{\Gamma}_{P}(\hat{t}, 1)\right), \\
-\Delta_{r} \hat{C} & =-\hat{\alpha} \hat{R}^{2} P \hat{C}
\end{aligned}
$$

with initial conditions $\hat{R}\left(\hat{t}^{0}\right)=\hat{R}^{0}$ and $P\left(\hat{t}^{0}, \cdot\right)=P^{0}(\cdot)$ at initial time $\hat{t}^{0}$ and boundary conditions $\left.\partial_{r} \hat{C}\right|_{r=0}=0$ and $\left.\hat{C}\right|_{r=1}=1$.

For brevity, we omit the carets on the dimensionless quantities in the following.

\subsection{Choice of the proliferation and switch rates}

The parameters of the non-dimension model of Section 2.3 are the following:

- the proliferation rate function $\gamma_{0}(C)$,

- the switch rate function $\gamma_{1}(C)$ from proliferating state to quiescent state, 
- the nutrient, growth factor, and oxygen consumption $\alpha$.

Regarding the calibration of the model parameters, we simplify the definitions of the functions $\gamma_{0}$ and $\gamma_{1}$ and we reduce the number of their parameters.

Choice of the proliferation rate. The proliferation rate $\gamma_{0}$ is assumed to be constant. This assumption is done in order to benefit from the formula of the radius given by (6b). Actually, assuming $\gamma_{0}$ constant leads to the following explicit relation between the radius and the total mass of proliferating cells:

$$
R(t)=R^{0} \exp \left(\gamma_{0} \int_{t^{0}}^{t} M_{P}(\tilde{t}) \mathrm{d} \tilde{t}\right),
$$

where $M_{P}$ denotes the total mass of proliferating cells at time $t$, defined by

$$
M_{P}(t)=\int_{0}^{1} P(t, r) r^{2} \mathrm{~d} r
$$

Regarding equation (9), the parameter $\gamma_{0}$ gives an important information on the average proliferation rate of proliferating cells. Indeed, from the first mean value theorem for definite integrals, we can interpret the constant value $\gamma_{0}$ as the mean value (in space and in time) of the proliferation rate. This mean value drives the increase of the spheroid volume and is independent of the model we choose for the diffusion and consumption of the nutrients, growth factor, and oxygen. Moreover, a first estimation of the parameter $\gamma_{0}$ can be obtained by using the formula satisfied by the radius $(9)$.

Choice of the switch rate. Based on first comparisons between the experimental data and the numerical simulations, we observed that the proliferation gradient can be reproduced by using only the right-hand side of a sigmoid function. Therefore, we have chosen the following definition for the switch rate $\gamma_{1}$ (see Appendix 8.3 for a detailed description of this choice):

$$
\gamma_{1}(C)=a(C-1)^{2 m}+\gamma_{1, C_{0}}
$$

where $a$ and $\gamma_{1, C_{0}}$ are in $[0,+\infty)$ and $m$ is a positive integer. The parameter $a$ drives the maximum value of $\gamma_{1}$ and the parameter $m$ drives the stiffness of $\gamma_{1}$. See Figure 2 for the simulation of this function $C \longmapsto \gamma_{1}(C)$. In the simulations presented in Section 4 , the parameter $m$ has been set to 3 . This value was found to be sufficient to obtain the qualitative behavior observed in the experimental data. In Appendix 8.4, we study numerically the sensitivity of the model with respect to the parameters $m, a$, and $\alpha$.

\section{Model calibration}

In this section, we present the method used for the model calibration. In the first paragraph, we present the data post-processing used for the comparisons between the data and the simulation of the model. The calibration algorithm is described in the second paragraph. The main part of this algorithm, which is the initialization of the model parameters, is detailed in the last paragraph.

Remark 3.1. In order to minimize the errors coming from the data, we prefer to translate the simulations on the 2D slices to compare simulations with the data, instead of transforming the $2 D$ slices of the experiments into reconstructed $3 D$ data. Such a translation dependes only on discretization errors, which can be controlled. 


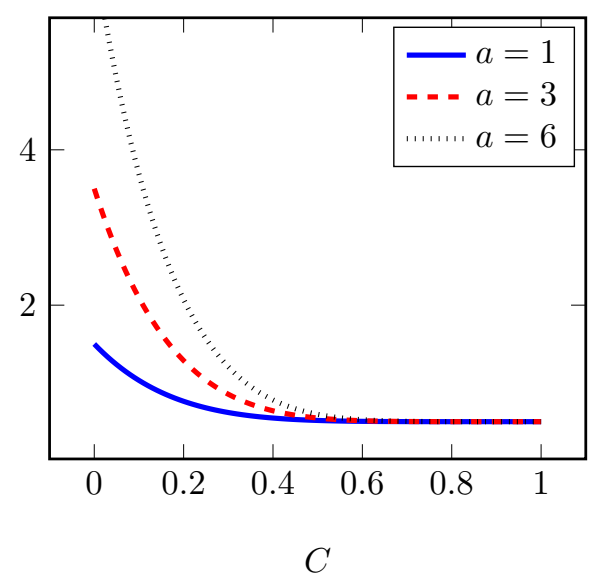

(a) Plot with $\gamma_{1, C_{0}}=0.5, m=3$, and several values of $a$.

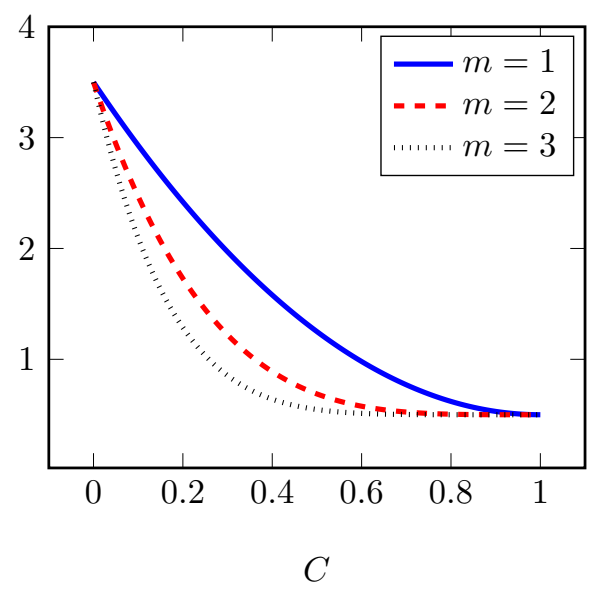

(b) Plot with $\gamma_{1, C_{0}}=0.5, a=3$, and several values of $m$.

Figure 2: Plot of the function $C \longmapsto \gamma_{1}(C)$ given by equation (11).

\subsection{Data post-processing}

The data used for computing the proliferating cells density consist of the DAPI images for staining the cell nuclei and the EdU images for staining the proliferating cell nuclei (see Figure 3). Briefly, images from spheroid sections were acquired by using an epifluorescence microscope. The nuclei were segmented based on DAPI fluorescence and EdU positive cells were identified by using a thresholding method. These pre-processing steps were done by using Cellomics Technologies software (Compartimental Bioapplication-Thermo Scientific). The experiments have been reproduced about 20 times for each time point and each experimental condition in order to reduce the measurement errors. The resulting variability observed in the data 33 . provide an estimate on the accuracy of the images. We refer to 33 for further details on the experimental setup and the method used to extract data from the experimental images.

After the pre-processing step, the resulting data consist of the repartition of cells, respectively the repartition of EdU positive cells, with respect to the cell distance to the surface of the spheroid, as shown on the left of Figure 4.

In this section, we present the method used to compare the distribution of proliferating cells obtained by the data to the density of proliferating cells given by the numerical simulation of the model. The main idea is to compute the fraction of proliferating cells on several regions of the spheroids (see Figure 4). For this purpose, we consider a partition $r_{1}=0<r_{2}<\cdots<r_{K+1}=1$ of the interval $[0,1]$, where $K$ is a non-negative integer, and for $k=1, \ldots, K$, we define the following distance class:

$$
L_{k}=\left(r_{k}, r_{k+1}\right) \text {. }
$$

For each cell of a spheroid from data, we compute its relative radial coordinate from its distance to the spheroid surface. For any $k=1, \ldots, K$, we denote by $\# P_{L_{k}}$, resp. $\# Q_{L_{k}}$, the number of proliferating cells, resp. quiescent cells, in the distance class $L_{k}$ given on the $2 \mathrm{D}$ section of the spheroid and we define the fraction of proliferating cells in the distance class $L_{k}$ as follows:

$$
\bar{P}_{L_{k}}=\frac{\# P_{L_{k}}}{\# P_{L_{k}}+\# Q_{L_{k}}} \text {. }
$$

This fraction of proliferating cells in the distance class $L_{k}$ can be easily computed from the distribution of proliferating cells and the total number of cells. 

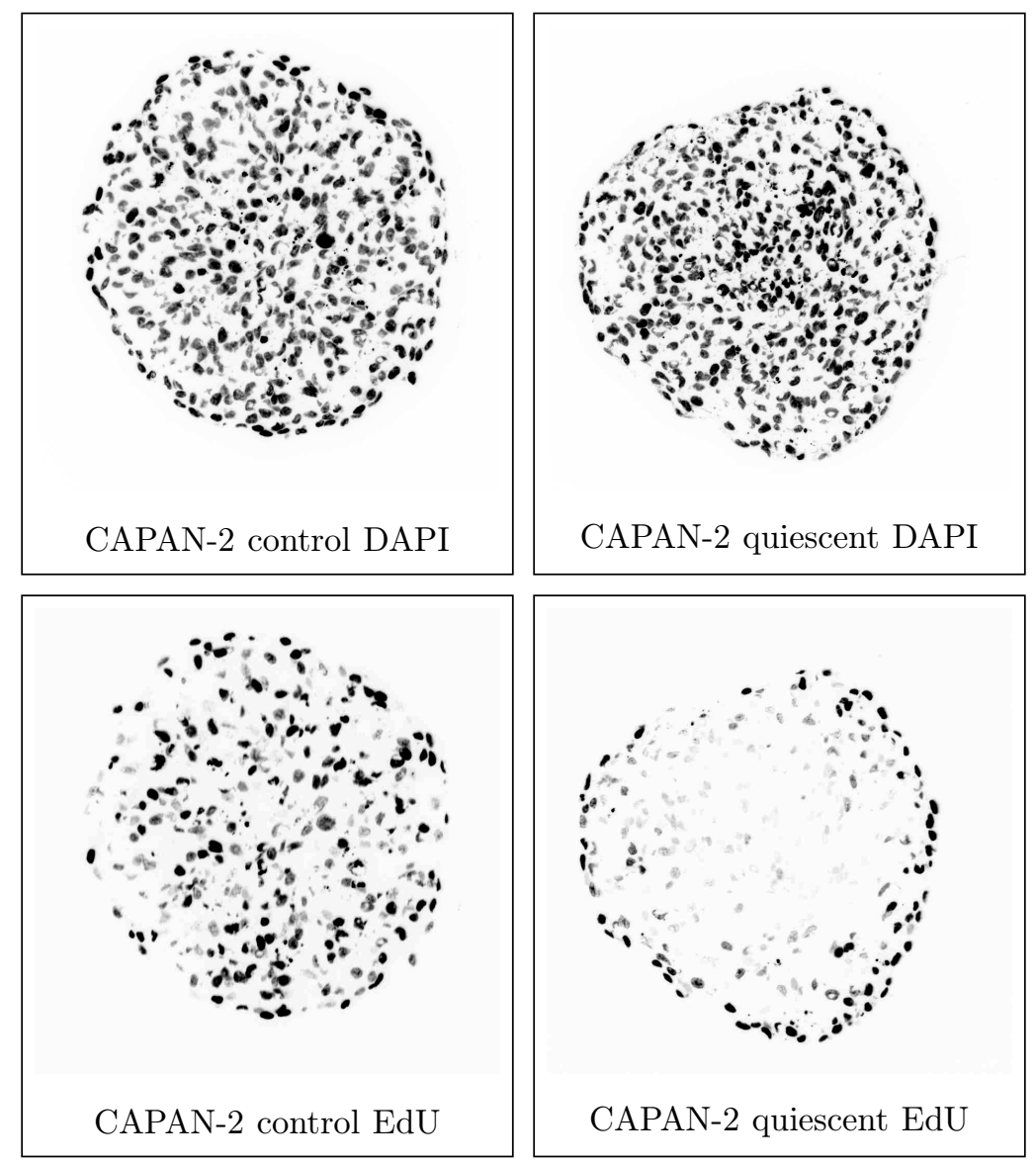

Figure 3: Images of cryosections of spheroids made with the tumor pancreatic cell line CAPAN-2 and grown in the presence of epidermal growth factor (EGF) (control, left) or in the absence of the growth factor (quiescent, right). Proliferating cells are visualized after a 24h-incorporation and immunodetection of the EdU marker (bottom). All nuclei are stained with DAPI (top).

Based on the data presented in Fig. 5, we make the approximation that the average area occupied by a proliferating cell is equal to the average area occupied by a quiescent cell. Although there are more proliferating cells in the CAPAN-2 control experiment than in the CAPAN-2 quiescent experiment (see Fig. 6), we do not observe a significant difference in the average area occupied by the cells (see Figure 5).

Proposition 3.2. Under the assumption that the average area occupied by a proliferating cell is equal to the average area occupied by a quiescent cell, the relation between the fraction $\bar{P}_{L_{k}}(t)$ and the proliferating cell density $P(t, \cdot)$ is the following:

$$
\bar{P}_{L_{k}}(t)=\frac{2}{r_{k+1}^{2}-r_{k}^{2}} \int_{r_{k}}^{r_{k+1}} P(t, r) r \mathrm{~d} r
$$

Proof. We denote by $A_{P}$, resp. $A_{Q}$, the average of the inverse of the proliferating cells number, resp. quiescent cells number, per area unit. These numbers correspond to the actual average area occupied by the proliferating and the quiescent cells, that is the cell area plus the surrounding space between the cells. We assume these quantities to be constant in space and time. Then the 


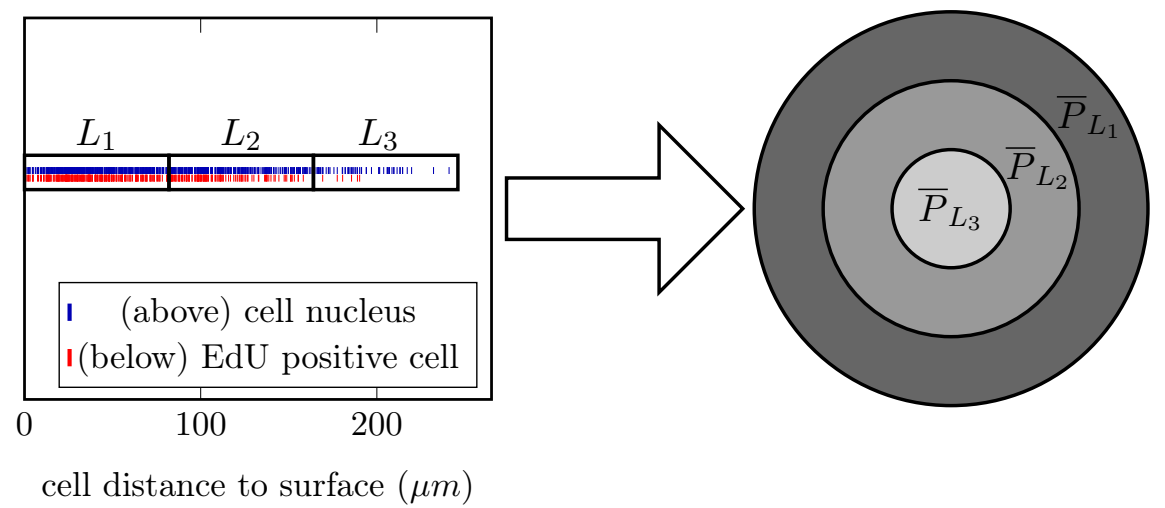

Figure 4: Schematic representation of the method used for the computation of proliferating cell proportion $\bar{P}_{L_{k}}$ on each distance class $L_{k}$. First, the distribution of cells and the distribution of EdU positive cells with respect to their distance to the spheroid surface are computed from the DAPI images and EdU images (see Figure 3). Then the cells are separated into several distances classes $L_{k}$ and the proportion of EdU positive cells is calculated in each class.

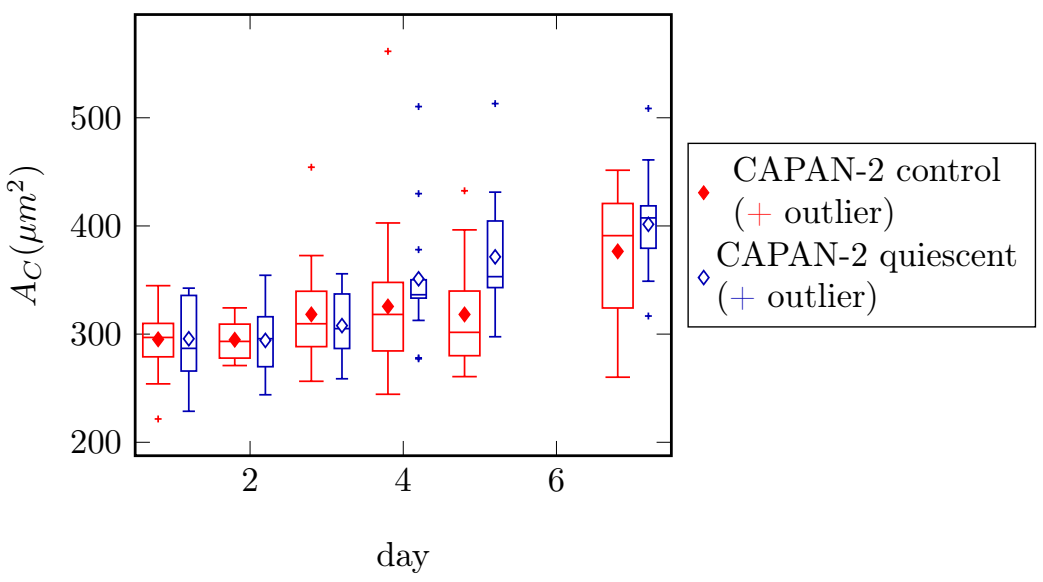

Figure 5: Average area $A_{C}$ occupied by a cell in CAPAN-2 experiments. Area is computed by dividing the spheroid area by the number of cells in the spheroid image.

area occupied by proliferating cells at time $t$ in the distance class $L_{k}$ for a spheroid is given by

$$
A_{P} \# P_{L_{k}}(t)=2 \pi R(t)^{2} \int_{r_{k}}^{r_{k+1}} P(t, r) r \mathrm{~d} r
$$

The area occupied by proliferating and quiescent cells in the class $L_{k}$ is obtained by integrating the saturation assumption (3) in space between $r_{k}$ and $r_{k+1}$ :

$$
A_{P} \# P_{L_{k}}(t)+A_{Q} \# Q_{L_{k}}(t)=2 \pi R(t)^{2} \frac{r_{k+1}^{2}-r_{k}^{2}}{2} .
$$

Therefore, the fraction of proliferating cells in the distance class $L_{k}$ is given by

$$
\frac{\# P_{L_{k}}(t)}{\# P_{L_{k}}(t)+\left(A_{Q} / A_{P}\right) \# Q_{L_{k}}(t)}=\frac{2}{r_{k+1}^{2}-r_{k}^{2}} \int_{r_{k}}^{r_{k+1}} P(t, r) r \mathrm{~d} r .
$$

We conclude by using the assumption $A_{Q}=A_{P}$. 
Remark 3.3. Equation (14) gives the projection of $3 D$ radial densities $P(t, \cdot)$ on the space of $2 D$ slices data. This formula is used in the calibration algorithm for translating the $3 D$ simulations into $2 D$ slices corresponding to the $2 D$ slices data.

From equation (14), we define the quantities which are used for the calibration of the model.

Definition 3.4. Let $N$ be the number of time points in the experimental data and let $t^{0}, \ldots, t^{N}$ be the corresponding time points. For any time $t \in\left\{t^{0}, \ldots, t^{N}\right\}$, we denote by $R^{D}(t)$ the mean radius and by $\bar{P}_{L_{k}}^{D}(t)$ the mean proportion of proliferating cells in $L_{k}$ in the data set of spheroids at time $t$. We define the piecewise constant function $P^{D}(t, \cdot)$ as follows:

$$
P^{D}(t, r)=\bar{P}_{L_{k}}^{D}(t), \quad \text { for } k \text { such that } r \in L_{k} .
$$

Remark 3.5. When the proliferating cell density $P(t, r)$ is a piecewise constant function, with constant values in the intervals $L_{k}$, equation (14) leads to $\bar{P}_{L_{k}}(t)=P(t, r)$ for $r$ in $L_{k}$. Therefore the above definition corresponds to the projection of the $2 D$ slices data on the space of piecewise constant radial functions with constant values in the intervals $L_{k}$.

\subsection{Calibration algorithm}

Based on the definition of the proliferation rate and the switch rate presented in Section 2.4. the model has 4 parameters to be calibrated: $\gamma_{0}, \gamma_{1, C_{0}}, a$, and $\alpha$. Hereafter for any parameters $\gamma_{0}, \gamma_{1, C_{0}}, a$, and $\alpha$, we denote by $\theta$ the vector $\left(\gamma_{0}, \gamma_{1, C_{0}}, a, \alpha\right)$.

Definition 3.6. Let $R^{D}$ and $P^{D}$ be as in Definition 3.4. For any vector of parameters $\theta=$ $\left(\gamma_{0}, \gamma_{1, C_{0}}, a, \alpha\right)$ and any time $t \in\left\{t^{0}, \ldots, t^{N}\right\}$, we define the following quantities:

- (initial conditions) we denote by $R^{0}=R^{D}\left(t^{0}\right)$ and by $P^{0}(\cdot)=P^{D}\left(t^{0}, \cdot\right)$,

- (simulation of the model) let $R^{\theta}(t)$ be the radius and $P^{\theta}(t, r)$ be the proliferating cell density given as the solution to the model (6) with initial conditions $R^{0}$ and $P^{0}$,

- (fraction of proliferating cells) for any $k=1, \ldots, K$ we define $\bar{P}_{L_{k}}^{\theta}(t)$ as follows:

$$
\bar{P}_{L_{k}}^{\theta}(t)=\frac{2}{r_{k+1}^{2}-r_{k}^{2}} \int_{r_{k}}^{r_{k+1}} P^{\theta}(t, r) r \mathrm{~d} r .
$$

By using Definition 3.4 and Definition 3.6, we define the discrepancy function used for the calibration of the model parameters.

Definition 3.7. For any parameters $\theta=\left(\gamma_{0}, \gamma_{1, C_{0}}, a, \alpha\right)$, the discrepancy between experimental data and the numerical simulations is defined as follows:

$$
\varepsilon^{2}(\theta)=\varepsilon_{R}^{2}(\theta)+\varepsilon_{P}^{2}(\theta),
$$

where $\varepsilon_{R}^{2}(\theta)$ denotes the normalized least square discrepancy for the radius:

$$
\varepsilon_{R}^{2}(\theta)=\frac{1}{N} \sum_{t \in\left\{t^{1}, \ldots, t^{N}\right\}}\left(\frac{R^{D}(t)-R^{\theta}(t)}{R^{D}(t)}\right)^{2},
$$

and $\varepsilon_{P}^{2}(\theta)$ denotes the least square discrepancy for the fraction of proliferating cells:

$$
\varepsilon_{P}^{2}(\theta)=\frac{1}{N} \sum_{t \in\left\{t^{1}, \ldots, t^{N}\right\}} \frac{1}{K} \sum_{k=1}^{K}\left(\bar{P}_{L_{k}}^{D}(t)-\bar{P}_{L_{k}}^{\theta}(t)\right)^{2} .
$$


The objective of the calibration is to find the optimal vector of parameters, denoted by $\theta$, which minimizes $\varepsilon^{2}$. The outline of the calibration algorithm is the following:

1. the first step is the initialization of the parameters of the model. In this step, the structure of the model is used in order to obtain a first approximation of the parameters, denoted by $\theta^{0}$. This initialization of the parameters is presented in Section 3.3 .

2. the second step is the optimization of the least square discrepancy functional defined in Definition 3.7 by using an iterative algorithm. This iterative algorithm is initialized with the first approximation of the parameters $\theta^{0}$. In this step, we use the optimization function scipy.optimize.minimize from the open source Python library SciPy [30] with the LBFGS-B algorithm [7, 51]. The function returns the optimal parameters denoted by $\theta$.

\subsection{Initialization of the model parameters}

In this section, we present the method used to get the first estimate for the parameters, denoted by $\theta^{0}=\left(\gamma_{0}^{0}, \gamma_{1, C_{0}}^{0}, a^{0}, \alpha^{0}\right)$. This estimate is obtained in three steps: we start by estimating $\gamma_{0}^{0}$, then we use this value to get $\gamma_{1, C_{0}}^{0}$, and we finally compute $a^{0}$ and $\alpha^{0}$ by using $\gamma_{0}^{0}$ and $\gamma_{1, C_{0}}^{0}$.

Initialization of $\gamma_{0}$. The value of the first estimate for $\gamma_{0}$ is computed by using the formula for the radius (6b). More precisely, we define the following objective function

$$
\mathcal{R}\left(\gamma_{0}\right)=\frac{1}{N} \sum_{t \in\left\{t^{1}, \ldots, t^{N}\right\}}\left[\frac{R^{D}(t)-R^{D}\left(t^{0}\right) \exp \left(\gamma_{0} \int_{t^{0}}^{t} \int_{0}^{1} P^{D}(\tilde{t}, r) r^{2} \mathrm{~d} r \mathrm{~d} \tilde{t}\right)}{R^{D}(t)}\right]^{2},
$$

where $R^{D}$ and $P^{D}$ are given in Definition 3.4 . Then we define $\gamma_{0}^{0}$ by

$$
\gamma_{0}^{0}=\underset{\gamma_{0}}{\arg \min } \mathcal{R}\left(\gamma_{0}\right) \text {. }
$$

This minimizer is estimated by using Newton algorithm.

Initialization of $\gamma_{1, C_{0}}$. In order to get the first estimate for $\gamma_{1, C_{0}}$, we use the formula (26) given in Appendix 8.1. This formula explicits the proliferating cell density at spheroid surface. We define the following objective function:

$$
\mathcal{P}\left(\gamma_{0}, \gamma_{1, C_{0}}\right)=\frac{1}{N} \sum_{t \in\left\{t^{1}, \ldots, t^{N}\right\}}\left[P^{D}(t, 1)-f(t) P^{D}\left(t^{0}, 1\right)\right]^{2},
$$

where $P^{D}$ is given in Definition 3.4 and

$$
f(t)=\frac{\exp \left(\left(\gamma_{0}-\gamma_{1, C_{0}}\right)\left(t-t^{0}\right)\right)}{1+\gamma_{0} \frac{\exp \left(\left(\gamma_{0}-\gamma_{1, C_{0}}\right)\left(t-t^{0}\right)\right)-1}{\gamma_{0}-\gamma_{1, C_{0}}}}
$$

Then we use the parameter $\gamma_{0}^{0}$ previously estimated and we define $\gamma_{1, C_{0}}^{0}$ by the following:

$$
\gamma_{1, C_{0}}^{0}=\underset{\gamma_{1, C_{0}}}{\arg \min } \mathcal{P}\left(\gamma_{0}^{0}, \gamma_{1, C_{0}}\right)
$$

This minimizer is estimated thanks to the standard Newton algorithm. 
Initialization of $a$ and $\alpha$. The first estimate for the parameters $a$ and $\alpha$ is obtained by using the previous estimates for $\gamma_{0}$ and $\gamma_{1, C_{0}}$ and by scanning the parameter space in log scale $(a$ and $\alpha$ are assumed to be positive). We define $\left(a^{0}, \alpha^{0}\right)$ as the minimizer of $\varepsilon^{2}\left(\gamma_{0}^{0}, \gamma_{1, C_{0}}^{0}, \cdot, \cdot\right)$ where $\varepsilon^{2}$ is given by Definition 3.7 .

\section{Results}

In this section, we present the results of the model calibration method developed in Section 3 . Then we compare the prediction of the model with some classical ODE models.

\subsection{Calibration results}

Here we present the results of the calibration of the model for four sets of data.

The first two sets of spheroid data consist of CAPAN-2 spheroids (CAPAN-2 cell line is composed of pancreatic tumor cells):

- CAPAN-2 control: this set is composed of spheroids grown in presence of the epidermal growth factor (EGF);

- CAPAN-2 quiescent: this set is composed of spheroids deprived of growth factor at Day 0 .

These two experiments have been repeated twice, with 12 spheroids for each time point and each experiments. Therefore, there are about 20 spheroids for each time point for both CAPAN-2 control and CAPAN-2 quiescent experiments. Moreover, about 20 spheroids were also grown continuously until Day 7 and without imaging for both CAPAN-2 control and CAPAN-2 quiescent experiments. These spheroids provide continuous data on the time evolution of the radius. Therefore they are used as data for the radius.

Remark 4.1. Due to the experimental protocol (images of spheroids cryosections), the data used for collecting the distribution of proliferating cells are not continuous in time. In this study, we assume that the evolution of the average values computed from a large number of spheroids (about 20 spheroids per time point) is continuous in time.

The two other sets of data consist of the analysis of cell proliferation on spheroids made of the colon carcinoma cell line HCT-116:

- HCT-116 21\% $\mathrm{O}_{2}$ : spheroids grown at $21 \%$ of oxygen, the concentration in the air;

- HCT-116 5\% $\mathrm{O}_{2}$ : these are spheroids grown at $5 \%$ of oxygen, corresponding to a more physiological concentration in tissues, or physioxia condition.

For these two sets, images are acquired at two times Day 3 and Day 6.

The data processing (presented in Section 3.1) of the four set of data presented above is done by choosing the number of distance classes $K=7$ and the subdivision $r_{1}=0<\cdots<r_{8}=1$ in the set

$$
\{0,0.20,0.34,0.46,0.58,0.68,0.84,1\} \text {. }
$$

The above choice was done to keep the balance between taking $K$ large (in order to get enough proportions $\bar{P}_{L_{k}}$ ) and taking the classes $L_{k}$ large (in order to compute the proportions with a 
large enough number of cells). For such a subdivision, we have at least 20 cells for almost every class and every spheroid. The results of the calibration for the CAPAN-2 spheroids and the HCT-116 spheroids are in given in Figure 6 and Figure 7 respectively. The parameters obtained by the calibration algorithm are given in Table 1. For the CAPAN-2 experiments, we have also tested the model ability to predict the evolution of the spheroid. For that purpose, we performed the calibration presented in Section 3 by using the CAPAN-2 experimental data from Day 1 to Day 5. Then we performed the simulation of the model from Day 1 to Day 7 (see the dashed lines in Figure 6). This provides the prediction of the spheroid evolution from Day 5 to Day 7. The simulation are plotted with dashed lines in Figure 6

\begin{tabular}{ccccccc}
\hline & & \multicolumn{2}{c}{ parameters } & discrepancy \\
\hline & & $\gamma_{0}$ & $\gamma_{1, C_{0}}$ & $a$ & $\alpha$ & $\varepsilon$ \\
\hline \multirow{2}{*}{ CAPAN-2 control } & fit & $3.58 \mathrm{e}-01$ & $1.37 \mathrm{e}-01$ & $2.07 \mathrm{e}+00$ & $2.40 \mathrm{e}+00$ & $7.18 \mathrm{e}-02$ \\
& prediction & $4.07 \mathrm{e}-01$ & $1.66 \mathrm{e}-01$ & $1.00 \mathrm{e}+05$ & $2.30 \mathrm{e}-01$ & $4.50 \mathrm{e}-02$ \\
\hline \multirow{2}{*}{ CAPAN-2 quiescent } & fit & $6.74 \mathrm{e}-01$ & $8.51 \mathrm{e}-01$ & $1.91 \mathrm{e}+00$ & $3.85 \mathrm{e}+01$ & $5.20 \mathrm{e}-02$ \\
& prediction & $6.66 \mathrm{e}-01$ & $7.91 \mathrm{e}-01$ & $1.71 \mathrm{e}+00$ & $4.80 \mathrm{e}+01$ & $5.54 \mathrm{e}-02$ \\
\hline HCT-116 21\% $\mathrm{O}_{2}$ & & $6.39 \mathrm{e}-01$ & $4.75 \mathrm{e}-02$ & $1.03 \mathrm{e}+00$ & $1.72 \mathrm{e}-03$ & $2.70 \mathrm{e}-02$ \\
\hline HCT-116 5\% $\mathrm{O}_{2}$ & & $3.19 \mathrm{e}-01$ & $5.95 \mathrm{e}-02$ & $8.06 \mathrm{e}-01$ & $2.75 \mathrm{e}-03$ & $3.36 \mathrm{e}-02$ \\
\hline
\end{tabular}

Table 1: Summary of the parameters obtained by minimizing the discrepancy $\varepsilon$ (defined in Definition 3.7) by using the calibration algorithm of Section 3 on the different datasets with the parameter $m$ set to 3. Regarding the CAPAN-2 datasets, the "prediction" is obtained by calibrating the model by using the data from Day 1 to Day 5. The corresponding simulations of the model are given in Figure 6 and Figure 7

\subsection{Comparison of the prediction with some ODE models}

In this section, we compare the prediction for tumor radius obtained by the model calibration on the CAPAN-2 data with some classical ODE models. The ODE models are defined by using the tumor volume $V$. Here, we rewrite them by using the tumor radius instead and we denote their parameters by $\theta=\left(\theta_{1}, \theta_{2}, \ldots\right)$. For simplicity, we write the models for $t^{0}=0 \mathrm{~s}$. We consider the following models:

1. (Logistic) The logistic model is one of the simplest model that account for growth saturation. It is defined by a linear decrease of the relative growth rate in proportion to the volume:

$$
\frac{d}{d t} V(t)=\theta_{2} V(t)\left(1-\frac{V(t)}{\theta_{1}}\right)
$$

where $V^{0}$ is the initial tumor volume. By solving the ODE and using the initial condition $R^{0}$, we obtain the following explicit formula for the tumor radius:

$$
R(t)=R^{0}\left(\frac{\theta_{1}}{V^{0}+\left(\theta_{1}-V^{0}\right) e^{-\theta_{2} t}}\right)^{1 / 3},
$$

where $V^{0}=(4 / 3) \pi\left(R^{0}\right)^{3}$ is the initial volume of the tumor. 

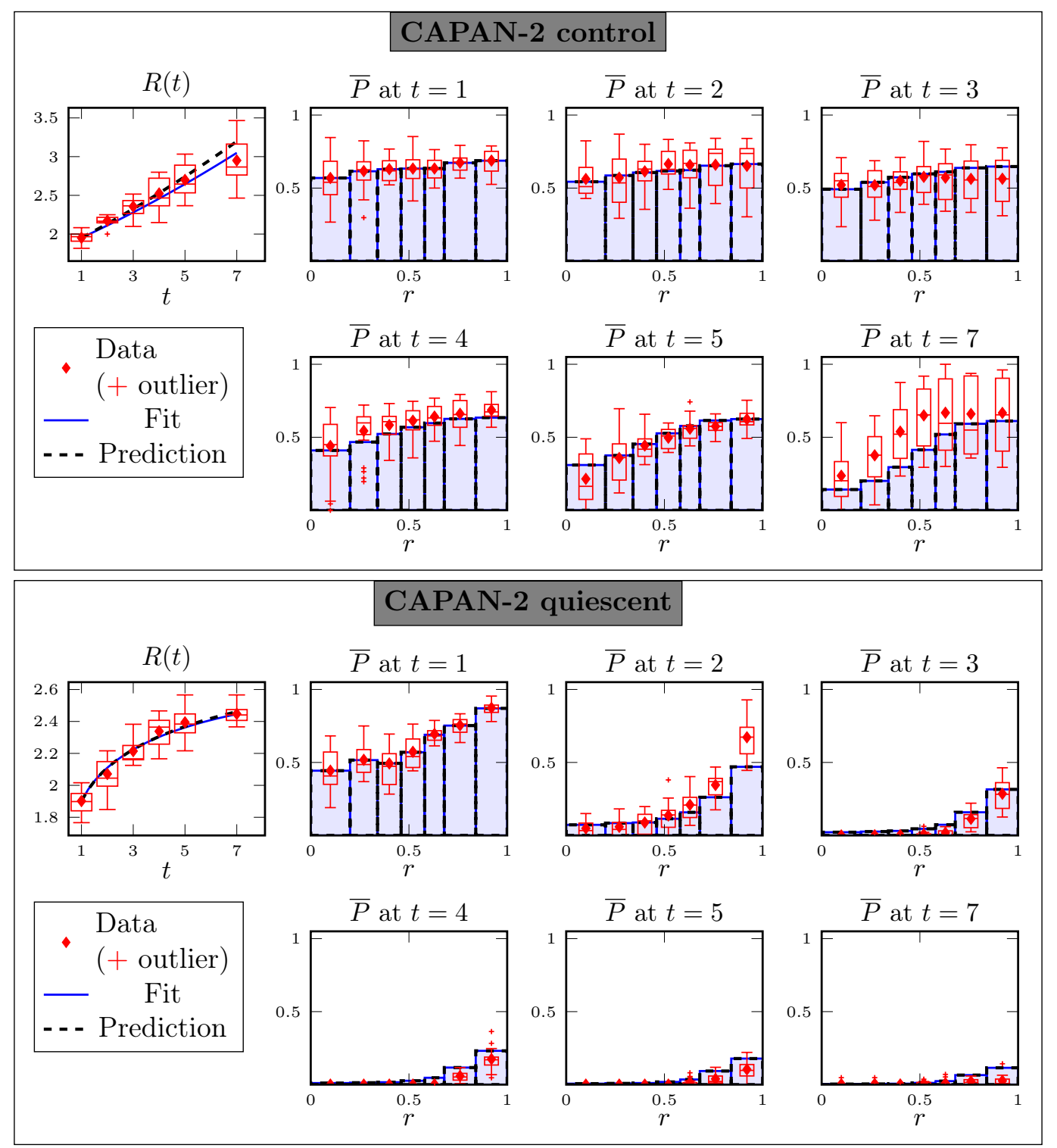

Figure 6: Comparisons between numerical simulations and experimental data for CAPAN-2 (pancreatic cancer cells) spheroids for two experiments. The fits to the data are obtained by minimizing the discrepancy $\varepsilon$ (defined in Definition 3.7) by using the calibration algorithm of Section 3 with the parameter $m$ set to 3. Regarding the data, $P$ denotes the proportion of proliferating cells between two consecutive relative radial coordinates $r_{k}<r_{k+1}$ in the $2 \mathrm{D}$ images, while it denotes the $2 \mathrm{D}$ integral of $P$ between $r_{k}$ and $r_{k+1}$ for the simulation (the relation between these two quantities is given in equation (14)). The "prediction" is obtained by calibrating the model by using the data from Day 1 to Day 5. 

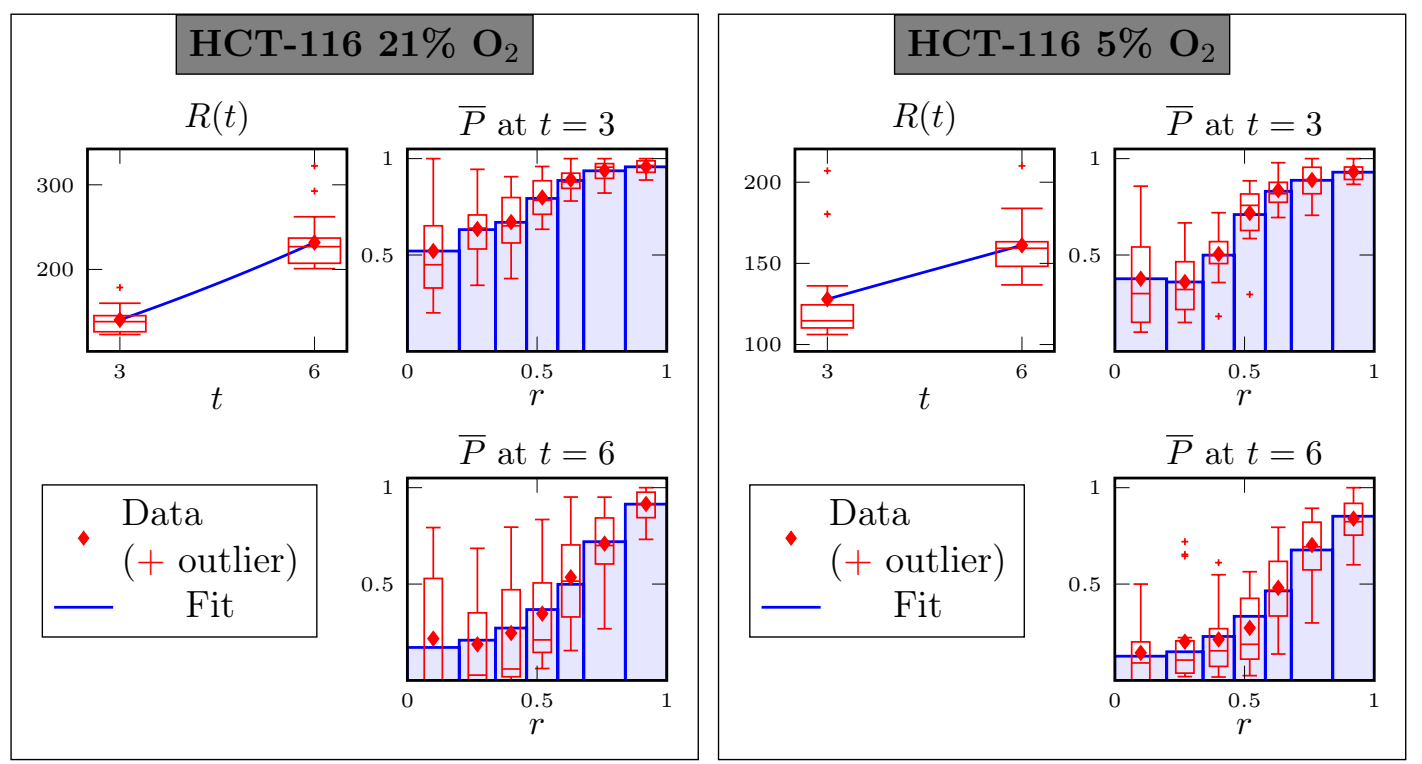

Figure 7: Comparisons between numerical simulations and experimental data for HCT-116 (colon cancer cells) spheroids for two experiments. The fits to the data are obtained by minimizing the discrepancy $\varepsilon$ (defined in Definition 3.7) by using the calibration algorithm of Section 3 with the parameter $m$ set to 3. Regarding the data, $P$ denotes the proportion of proliferating cells between two consecutive relative radial coordinates $r_{k}<r_{k+1}$ in the 2D images, while it denotes the 2D integral of $P$ between $r_{k}$ and $r_{k+1}$ for the simulation (the relation between these two quantities is given in equation (14)).

2. (Gompertz) The gompertz model is often used to model the growth of tumors. In this model, the decrease of the relative growth rate is exponential in time:

$$
\frac{d}{d t} V(t)=\theta_{1} e^{-\theta_{2} t} V(t)
$$

Solving the ODE leads to the following formula for the radius:

$$
R(t)=R^{0} \exp \left(\frac{1}{3} \theta_{1} \frac{1-e^{-\theta_{2} t}}{\theta_{2}}\right)
$$

3. (Reduced) We also consider the reduced model that is derived from our model under the following conditions (see Proposition 8.6 in Appendix 8.1):

- there is no proliferation gradient in the initial tumor (i.e the function $P^{0}$ is constant), - $a=0$ or $\alpha=0$ so that $\gamma_{1}(C)=\gamma_{1, C_{0}}$ is constant.

This model has only two parameters $\theta_{1}=\gamma_{0} P^{0}$ and $\theta_{2}=\gamma_{1, C_{0}}-\gamma_{0}$.

$$
R(t)=R^{0}\left(1+\theta_{1} \frac{\left(1-e^{-\theta_{2} t}\right)}{\theta_{2}}\right)^{1 / 3}
$$

It is worth nothing that the Taylor expansion of the functions $u \longmapsto \exp (u / 3)$ and $u \longmapsto$ $(1+u)^{1 / 3}$ coincide until order 1 so that the gompertz model and the reduced model are very close in the earlier times. 
The equations and solutions for the logistic model and the gompertz model can be found in the review [5]

For each model, we estimate the optimal parameters $\theta$ by minimizing the error for the radius $\varepsilon_{R}$ (as defined in Definition 3.4) by using the data from Day 1 to Day 5. Here again we use the optimization function scipy.optimize.minimize from the open source Python library SciPy [30] with the L-BFGS-B algorithm. Then we compare the results by estimating the following error:

$$
\varepsilon_{R}^{N}(\theta)=\left|\frac{R^{D}\left(t^{N}\right)-R^{\theta}\left(t^{N}\right)}{R^{D}\left(t^{N}\right)}\right|,
$$

where $t^{N}$ denotes the latest time, $R^{D}$ is defined in Definition 3.4 and $R^{\theta}$ denotes the model simulation for the optimal parameter $\theta$. The results are given in Figure 8 .
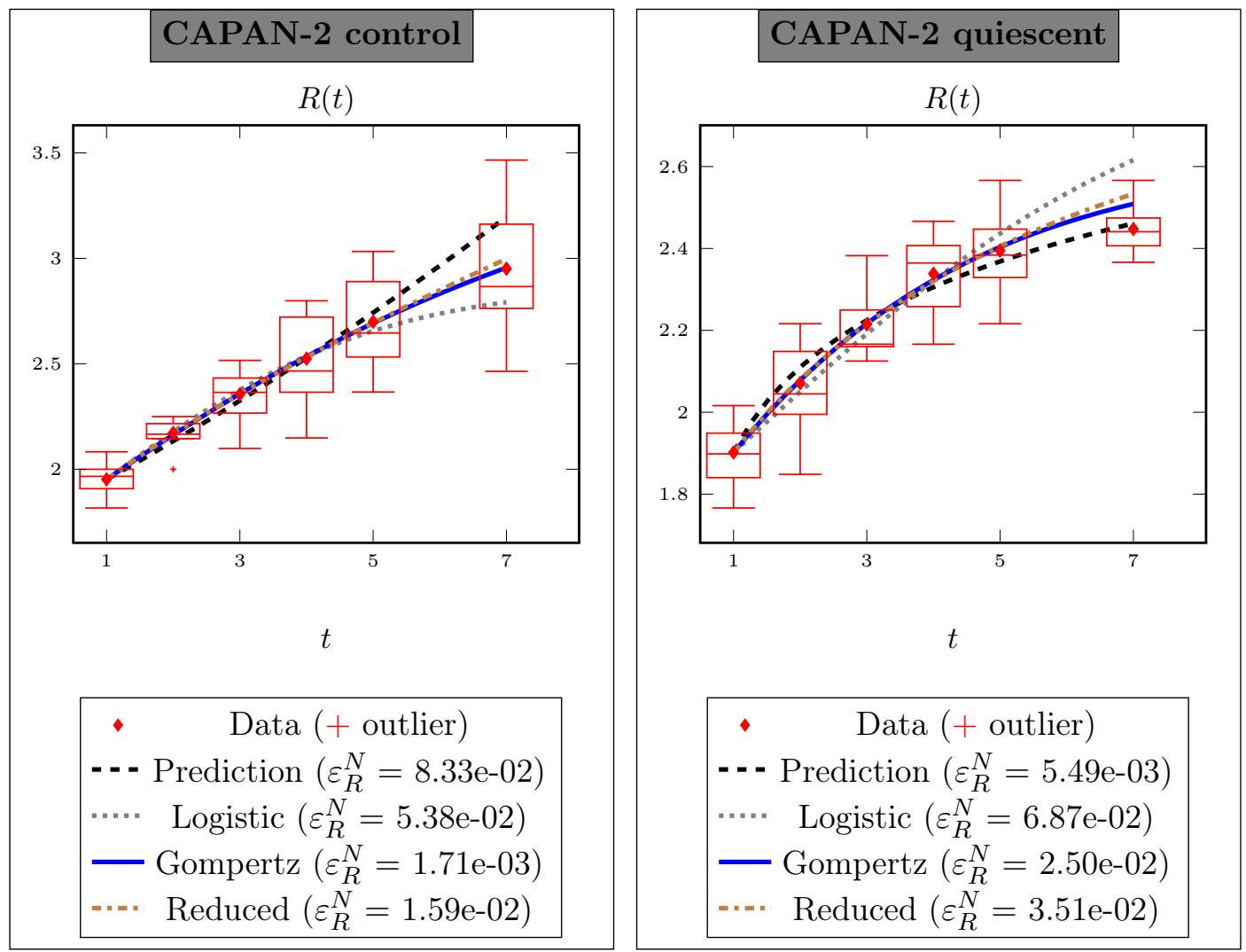

Figure 8: Comparison of the model prediction for the tumor radius with the ODE models defined in Section 4.2 Each model has been calibrated by using the data from Day 1 to Day $5 . \varepsilon_{R}^{N}$ is the relative error for the prediction of the last radius (defined in equation (25)).

Regarding the CAPAN-2 control data, we observe that the model prediction is not as accurate as the prediction obtained by the gompertz model or by the reduced model. The model loses accuracy while trying to fit the spatial data while the reduced model is able to give a better prediction for the linear regime. In this case, it seems that it should be better to assume no proliferation gradient. Regarding the CAPAN-2 quiescent data, the model is able to give the best prediction, although the fit to the data from Day 1 to Day 5 is the worst. In this case, the spatial data seems to improve the accuracy of the prediction in when the tumor growth reaches the sub-linear regime. 


\section{Discussion}

We built a model to describe the evolution and spatial structure of tumor spheroids submitted to different experimental condition. The model is based on the assumption that the diffusion of nutrients, growth factor, and oxygen inside a spheroid drives the creation of a proliferation gradient. Some of the previous models focused on the growth of the spheroid without accounting for the spatial tumor heterogeneity [36] and other previous models focused on this heterogeneity without accounting for tumor growth [26]. On the contrary, our model is able to describe these two aspects and their combined effects on the evolution of tumor spheroids. In [26], the authors focused on modeling the oxygen consumption within a spheroid and the extents of the necrotic core, hypoxic region, and proliferating rim. By contrast with the data used in [26], our experiments show that the transition between the proliferating rim and the quiescent core is not so sharp. Proliferating and quiescent cells are not homogeneously distributed, leading to a gradient of their distribution (see the spheroid images in Figure 3 or the distribution of proliferating cells in radial coordinates in Figure 6 and Figure 7). Our model describes the evolution of a mixture of two populations of cells. It is thus well suited for the experimental set-up of the present study. In particular, it is able to qualitatively reproduce the dynamics of the radius and the fraction of proliferating cells observed in 4 data sets (Figure 6 and Figure 7) by using much fewer parameters than the model proposed by Jagiella et al. [29]. In particular, our model reproduces the growth of tumor spheroid under tumor growth promoting conditions, such as high EGF (Epidermal Growth Factor) concentration for the CAPAN-2 control set or high oxygen concentration for the HCT-116 $21 \% \mathrm{O}_{2}$, as well as under physioxia conditions or low EGF concentration. Thus it reproduces the effect of both EGF concentration (for the CAPAN-2 experiments) and oxygen concentration (for the HCT-116 experiments). However, the variable $C$ is the combination of the concentration of several factors that promote cell proliferation, such as nutrients, growth factor, and oxygen. Therefore this variable cannot be explicitly computed from the experimental conditions.

A fit to the data shows that our model can explain the formation of a proliferation gradient but it is worth noting that this model is also able to predict the future evolution of the spheroid by knowing the previous evolution. By using the experimental data for the CAPAN-2 cells, we have also tested the ability of the model to predict the last time point by knowing the previous time points. The predictions for the proliferating cell distribution in the CAPAN-2 control and quiescent sets are similar to the simulations obtained by fitting the data from Day 1 to Day 7 (Figure 6). Thus the model is able to capture the dynamics of the proliferating cell distribution. Moreover, we also compared the prediction for the radius with the prediction obtained by classical ODE models such as the logistic model and gompertz model. This comparison shows that the use of the spatial data leads to a better accuracy when the gradient of proliferation is high enough (CAPAN-2 quiescent data). However, the accuracy is decreased by using the spatial data when the proliferation gradient is small (CAPAN-2 control data) and the comparison suggests to use the reduced ODE model derived from our model in such a case.

We eventually mention that a numerical study of the sensitivity of the model suggests that some parameters are correlated (see Appendix 8.4)). Therefore a forthcoming work will consist of reducing the number of parameters. In fact, during the space scanning which is done for the initialization step of the parameters $\alpha$ and $a$ (with fixed value for $m$ ), we observe a valley of values $(\alpha, a)$ such that the objective function is close to its minimum (Figure 11 in Appendix 8.4). In this valley, the simulations are very close to each other for $a$ varying from $10^{-1}$ to more than $10^{5}$ and for $\alpha$ varying from less than $10^{-1}$ to more than $10^{2}$. We recall that the parameter $\alpha$ drives the variation of the concentration $C$ of nutrients, growth factors, and oxygen in the spheroid, while the parameter $a$ drives the variation of the switch rate with respect to the concentration 
$C$. Thus increasing $a$ in the model can be compensated by decreasing $\alpha$. We have also studied the effect of the parameter $m$ on the optimal solution. Changing this value has an effect on the optimal value for the parameters $a$ and $\alpha$ (see Table 2 in Appendix 8.4). As observed in Appendix 8.4, the range for the parameters $a$ and $\alpha$ can vary markedly with $m$. Our intuition is that the qualitative behavior of the function $(t, r) \longmapsto \gamma_{1}(C(t, r))$ needed to fit the experimental proliferation gradient can be approximated by a wide subspace of the parameter space for $m, a$, and $\alpha$. However, a complete sensitivity analysis is far from the scope of the present paper.

\section{Conclusion}

We developed a partial differential equations model to understand the mechanisms involved in the apparition of a proliferation gradient in a MultiCellular Tumor Spheroid. The model is built on the basis of the observation of experimental data on spheroids growth and cell proliferation regionalization. These data provide a quantitative information on the distribution of proliferating and quiescent cells inside spheroids growing in an unconstrained medium. We proposed a method to compare these discrete data with the continuous cell densities. By using the radial symmetry observed in the data, we wrote the model in a way such that a first approximation of the two main parameters that drive the growth of the spheroid and the proliferation gradient can be directly estimated from the available data. These first estimations were obtained without running the whole model. Then we performed a full calibration of the model parameters on four sets of experimental data. The results of this calibration show that the proliferation gradient can be explained by using a simple relation between the concentration of growth factors and the rate at which cells enter the quiescent state. Moreover, the model is also able to give a prediction for the radius and the proliferation gradient of the last time point by knowing the previous time points. The model equations and the parameters obtained from the calibration can be used to build more complex models describing several properties of the spheroids, such as the effect of drugs or the mechanics of spheroid. For instance, the mechanical properties of spheroids have been studied in [13, 15] on the basis of the present work. Increasing progressively the complexity of the model could improve our understanding of the core properties of solid tumors and could lead to finding new therapeutic strategies.

\section{Acknowledgements}

This work was supported by ITMO Cancer AVIESAN (Alliance Nationale pour les Sciences de la Vie et de la Sante, National Alliance for Life Sciences \& Health) within the framework of the Plan Cancer and la Ligue Contre le Cancer (comite de la Haute-Garonne). The authors wish to acknowledge the TRI-Genotoul and ITAV imaging facilities.

C.P. is partly supported by the Plan Cancer projects DYNAMO (PC2015-15) and NUMEP (PC2016-15). This study has been carried out within the scope of the European Associate Lab EBAM, and the Inria Associate Team Num4SEP. T.M. is supported by the JSPS KAKENHI Grant Number JP16H06576.

The authors wish to acknowledge Pierre Weiss and Bernard Ducommun for fruitful discussions.

\section{References}

[1] T. Alarcón, H. M. Byrne, And P. K. Maini, A cellular automaton model for tumour 
growth in inhomogeneous environment, Journal of theoretical biology, 225 (2003), pp. 257274 .

[2] D. Ambrosi And L. Preziosi, On the closure of mass balance models for tumor growth, Mathematical Models and Methods in Applied Sciences, 12 (2002), pp. 737-754.

[3] A. R. Anderson And M. Chaplain, Continuous and discrete mathematical models of tumor-induced angiogenesis, Bulletin of mathematical biology, 60 (1998), pp. 857-899.

[4] Y. H. Bae, R. J. Mrsny, and K. Park, Cancer targeted drug delivery, 2013.

[5] S. Benzekry, C. Lamont, A. Beheshti, A. Tracz, J. M. Ebos, L. Hlatky, and P. HAhnfeldt, Classical mathematical models for description and prediction of experimental tumor growth, PLoS Comput Biol, 10 (2014), p. e1003800.

[6] D. Bresch, T. Colin, E. Grenier, B. Ribba, and O. Saut, A viscoelastic model for avascular tumor growth, Discrete and Continuous Dynamical Systems Supplements, 2009 (2009), pp. 101-108.

[7] R. H. Byrd, P. Lu, J. Nocedal, And C. Zhu, A limited memory algorithm for bound constrained optimization, SIAM Journal on Scientific Computing, 16 (1995), pp. 1190-1208.

[8] H. Byrne And M. A. Chaplain, Free boundary value problems associated with the growth and development of multicellular spheroids, European Journal of Applied Mathematics, 8 (1997), pp. 639-658.

[9] H. Byrne And D. Drasdo, Individual-based and continuum models of growing cell populations: a comparison, Journal of mathematical biology, 58 (2009), pp. 657-687.

[10] H. Byrne And L. Preziosi, Modelling solid tumour growth using the theory of mixtures, Mathematical Medicine and Biology, 20 (2003), pp. 341-366.

[11] J. Casciari, S. Sotirchos, And R. Sutherland, Mathematical modelling of microenvironment and growth in EMT6/Ro multicellular tumour spheroids, Cell proliferation, 25 (1992), pp. 1-22.

[12] X. Chen, S. Cui, And A. Friedman, A hyperbolic free boundary problem modeling tumor growth: Asymptotic behavior, Transactions of the American Mathematical Society, 357 (2005), pp. 4771-4804.

[13] T. Colin, G. Dechriste, J. Fehrenbach, L. Guillaume, V. Lobjois, and C. PoIGNARD, Experimental estimation of stored stress within spherical microtissues - what can and cannot be inferred from cutting experiments, under review, (2018).

[14] E. De Angelis And L. Preziosi, Advection-diffusion models for solid tumour evolution in vivo and related free boundary problem, Mathematical Models and Methods in Applied Sciences, 10 (2000), pp. 379-407.

[15] G. Dechriste, J. Fehrenbach, E. Griseti, V. Lobjois, and C. Poignard, Viscoelastic modeling of the fusion of multicellular tumor spheroids in growth phase, To appear in Journal of Theoretical Biology, (2018).

[16] M. Deville, R. Natalini, And C. Poignard, A continuum mechanics model of enzymebased tissue degradation in cancer therapies, under review, (2018). 
[17] S. Dormann And A. Deutsch, Modeling of self-organized avascular tumor growth with a hybrid cellular automaton, In silico biology, 2 (2002), pp. 393-406.

[18] D. Drasdo And S. Höhme, Individual-based approaches to birth and death in avascular tumors, Mathematical and Computer Modelling, 37 (2003), pp. 1163-1175.

[19] D. Drasdo And S. Höhme, A single-cell-based model of tumor growth in vitro: monolayers and spheroids, Physical biology, 2 (2005), p. 133.

[20] J. Folkman and M. Hochberg, Self-regulation of growth in three dimensions, The Journal of experimental medicine, 138 (1973), pp. 745-753.

[21] J. Freyer And R. Sutherland, A reduction in the in situ rates of oxygen and glucose consumption of cells in EMT6/Ro spheroids during growth, Journal of cellular physiology, 124 (1985), pp. 516-524.

[22] A. Friedman, A hierarchy of cancer models and their mathematical challenges, Discrete and Continuous Dynamical Systems Series B, 4 (2004), pp. 147-160.

[23] R. A. Gatenby and E. T. Gawlinski, A reaction-diffusion model of cancer invasion, Cancer research, 56 (1996), pp. 5745-5753.

[24] A. Gomes, L. Guillaume, D. R. Grimes, J. Fenrenbach, V. Lobjois, and B. Ducommun, Oxygen partial pressure is a rate-limiting parameter for cell proliferation in $3 d$ spheroids grown in physioxic culture condition, PloS one, 11 (2016), p. e0161239.

[25] H. Greenspan, On the growth and stability of cell cultures and solid tumors, Journal of theoretical biology, 56 (1976), pp. 229-242.

[26] D. R. Grimes, C. Kelly, K. Bloch, and M. Partridge, A method for estimating the oxygen consumption rate in multicellular tumour spheroids, Journal of The Royal Society Interface, 11 (2014), p. 20131124.

[27] P. D. W. Hamer, A. Van Tilborg, P. Eijk, P. Sminia, D. Troost, C. Van NoORDEn, B. Ylstra, AND S. LeEnSTRA, The genomic profile of human malignant glioma is altered early in primary cell culture and preserved in spheroids, Oncogene, 27 (2008), pp. 2091-2096.

[28] F. Hirschhaeuser, H. Menne, C. Dittfeld, J. West, W. Mueller-Klieser, and L. A. Kunz-Schughart, Multicellular tumor spheroids: an underestimated tool is catching up again, Journal of biotechnology, 148 (2010), pp. 3-15.

[29] N. Jagiella, B. Müller, M. Müller, I. E. Vignon-Clementel, and D. Drasdo, Inferring growth control mechanisms in growing multi-cellular spheroids of NSCLC cells from spatial-temporal image data, PLoS Comput Biol, 12 (2016), p. e1004412.

[30] E. Jones, T. Oliphant, P. Peterson, et Al., SciPy: Open source scientific tools for Python, 2001-.

[31] Y. Kim, M. A. Stolarska, And H. G. Othmer, A hybrid model for tumor spheroid growth in vitro $i$ : theoretical development and early results, Mathematical Models and Methods in Applied Sciences, 17 (2007), pp. 1773-1798. 
[32] L. A. Kunz-Schughart, J. P. Freyer, F. Hofstaedter, and R. Ebner, The use of 3-D cultures for high-throughput screening: the multicellular spheroid model, Journal of biomolecular screening, 9 (2004), pp. 273-285.

[33] J. Laurent, C. Frongia, M. Cazales, O. Mondesert, B. Ducommun, and V. LobJOIS, Multicellular tumor spheroid models to explore cell cycle checkpoints in 3D, BMC cancer, 13 (2013), p. 73.

[34] G. Lefebrre, F. Cornelis, P. Cumsille, T. Colin, C. Poignard, and O. Saut, Spatial modelling of tumour drug resistance: the case of GIST liver metastases, Mathematical Medicine and Biology, (2016).

[35] R.-Z. Lin And H.-Y. Chang, Recent advances in three-dimensional multicellular spheroid culture for biomedical research, Biotechnology journal, 3 (2008), pp. 1172-1184.

[36] D. Loessner, J. A. Flegg, H. M. Byrne, J. Clements, and D. Hutmacher, Growth of confined cancer spheroids: a combined experimental and mathematical modelling approach, Integrative Biology, 5 (2013), pp. 597-605.

[37] F. Pampaloni, E. G. Reynaud, and E. H. Stelzer, The third dimension bridges the gap between cell culture and live tissue, Nature reviews Molecular cell biology, 8 (2007), pp. 839-845.

[38] G. Pettet, C. Please, M. Tindall, and D. McElwain, The migration of cells in multicell tumor spheroids, Bulletin of mathematical biology, 63 (2001), pp. 231-257.

[39] K. Pietras, A. Östman, M. Sjöquist, E. Buchdunger, R. K. Reed, C.-H. Heldin, AND K. RUBIN, Inhibition of platelet-derived growth factor receptors reduces interstitial hypertension and increases transcapillary transport in tumors, Cancer research, 61 (2001), pp. 2929-2934.

[40] K. A. Rejniak and A. R. Anderson, Hybrid models of tumor growth, Wiley Interdisciplinary Reviews: Systems Biology and Medicine, 3 (2011), pp. 115-125.

[41] B. Ribba, O. Saut, T. Colin, D. Bresch, E. Grenier, and J.-P. Boissel, A multiscale mathematical model of avascular tumor growth to investigate the therapeutic benefit of anti-invasive agents, Journal of theoretical biology, 243 (2006), pp. 532-541.

[42] T. Roose, S. J. Chapman, And P. K. Maini, Mathematical models of avascular tumor growth, Siam Review, 49 (2007), pp. 179-208.

[43] G. Schaller and M. Meyer-Hermann, Multicellular tumor spheroid in an off-lattice Voronoi-Delaunay cell model, Physical Review E, 71 (2005), p. 051910.

[44] — Continuum versus discrete model: a comparison for multicellular tumour spheroids, Philosophical Transactions of the Royal Society of London A: Mathematical, Physical and Engineering Sciences, 364 (2006), pp. 1443-1464.

[45] J. A. Sherratt And M. A. Chaplain, A new mathematical model for avascular tumour growth, Journal of mathematical biology, 43 (2001), pp. 291-312.

[46] R. M. Sutherland, Cell and environment interactions in tumor microregions: the multicell spheroid model, Science, 240 (1988), p. 177. 
[47] K. R. Swanson, C. Bridge, J. Murray, and E. C. Alvord, Virtual and real brain tumors: using mathematical modeling to quantify glioma growth and invasion, Journal of the neurological sciences, 216 (2003), pp. 1-10.

[48] D. Wallace And X. Guo, Properties of tumor spheroid growth exhibited by simple mathematical models, Frontiers in oncology, 3 (2013), p. 51.

[49] J. P. WARD And J. R. King, Mathematical modelling of avascular-tumour growth, Mathematical Medicine and Biology, 14 (1997), pp. 39-69.

[50] _ Mathematical modelling of drug transport in tumour multicell spheroids and monolayer cultures, Mathematical biosciences, 181 (2003), pp. 177-207.

[51] C. Zhu, R. H. Byrd, P. Lu, and J. Nocedal, Algorithm 778: L-BFGS-B: Fortran subroutines for large-scale bound-constrained optimization, ACM Transactions on Mathematical Software (TOMS), 23 (1997), pp. 550-560. 


\section{Appendix}

\subsection{Properties of the model}

In this section, we give several properties satisfied by the solution of the model (6). Before writing the properties, we make the following assumptions:

- the proliferation rate is an non-decreasing $\mathcal{C}^{\infty}$ function of $C$, that is $\gamma_{0}^{\prime}(\cdot) \geq 0$;

- the switch rate is a non-increasing $\mathcal{C}^{\infty}$ function of $C$, that is $\gamma_{1}^{\prime}(\cdot) \leq 0$;

- the initial radius of the spheroid $R^{0}$ is non-negative;

- $P^{0}(\cdot)$ is a density, that is $P^{0} \in[0,1]$, is a $\mathcal{C}^{1}$ function and is a non-negative gradient of proliferation, that is $\partial_{r} P^{0} \geq 0$;

- the external concentration $C_{0}$ and the consumption rate $\alpha$ are positive.

We admit here that there exists a unique solution to the model (6) and this solution is smooth (this can be proved by a Banach contraction mapping theorem). Under the above assumptions, we can prove the following propositions. Proposition 8.1 states that $P$ is a density. The second proposition gives a formula for the cell density on the spheroid surface. This formula is used to initialize the parameter $\gamma_{1, C_{0}}$ in Section 3 . Such initialization is based on the choice of the formula (11) for $\gamma_{1}(C)$. In Proposition 8.3, we exhibit a maximum principle for the concentration $C$. As a consequence, Corollary 8.5 shows that the distribution of proliferating cells is increasing in the radial direction, as expected. As a conclusion, we give a bound on the radial advection velocity $v$ in Proposition 8.7. Such a bound provides a useful Courant-Friedrichs-Lewy (CFL) condition for the numerical simulation of the model.

For the sake of conciseness, the following propositions are given for $t^{0}=0$.

Proposition 8.1 ( $P$ is a density). For any $t \geq 0$ and $r \in[0,1]$ we have:

$$
P(t, r) \in[0,1] \text {. }
$$

Proof. We deduce the result by using the characteristic curves defined by the velocity field $v$, the assumption on the initial condition, and the fact that the right-hand side of equation (6c) vanishes for $P=0$ and is non-positive for $P=1$.

Proposition 8.2 (Proliferating cell density at spheroid surface). Denote by $\beta=\gamma_{1}\left(C_{0}\right)-\gamma_{0}\left(C_{0}\right)$. The proliferating cell density at the spheroid surface $P(t, 1)$ satisfies:

$$
P(t, 1)=\frac{e^{-\beta t}}{1+\gamma_{0}\left(C_{0}\right) P^{0}(1) \frac{1-\exp (-\beta t)}{\beta}} P^{0}(1) .
$$

Proof. Based on the fact that $v$ vanishes at $r=1$, the cell density at $r=1$ satisfies a logistic equation. The above formula is the explicit solution of this logistic equation.

Proposition 8.3 (Maximum principle for $C$ ). For any $t \geq 0$, let

$$
\begin{aligned}
P_{\min }(t) & =\min _{r \in[0,1]} P(t, r), \\
P_{\max }(t) & =\max _{r \in[0,1]} P(t, r) .
\end{aligned}
$$


For $m \in\{\min , \max \}$, let $C_{P_{m}}$ be the solution to the following equation

$$
-\Delta_{r} C_{P_{m}}=-\alpha R^{2} P_{m} C_{P_{m}}, \quad \text { with }\left.C_{P_{m}}\right|_{r=0}=C_{0} \text { and }\left.\partial_{r} C_{P_{m}}\right|_{r=0}=0,
$$

then $C_{P_{m}}$ is given by

$$
C_{P_{m}}(t, r)=\frac{C_{0}}{r} \frac{\sinh \left(r \sqrt{\alpha P_{m}(t) R(t)^{2}}\right)}{\sinh \left(\sqrt{\alpha P_{m}(t) R(t)^{2}}\right)},
$$

and $C$ satisfies the following inequality

$$
C_{P_{\max }}(t, r) \leq C(t, r) \leq C_{P_{\min }}(t, r) .
$$

Proof. The explicit formula for $C_{P_{m}}$ is obtained by using a power expansion in space of $C_{P_{m}}$, that is

$$
C_{P_{m}}(t, r)=\sum_{n \in \mathbb{N}} c_{n}(t) r^{n}
$$

and solving the recurrence relation obtained for each coefficient $c_{n}$ by using equation 6e .

To prove the inequality $C_{P_{\max }} \leq C$, we denote by $c=C-C_{P_{\max }}$. By using the assumptions and the non-negativity of $C_{P_{\max }}$, we obtain the following inequality

$$
-\Delta_{r} c+\alpha R^{2} P c \geq 0
$$

By multiplying this inequality by $c^{-}=\min (c, 0) \leq 0$ and integrating by parts in radial coordinates, we obtain the following (by using the facts that $\partial_{r} c \partial_{r} c^{-}=\left(\partial_{r} c^{-}\right)^{2}$ and $\left.c c^{-}=\left(c^{-}\right)^{2}\right)$ :

$$
\int_{0}^{1}\left(\partial_{r} c^{-}\right)^{2} r^{2} \mathrm{~d} r+\alpha R^{2} \int_{0}^{1} P\left(c^{-}\right)^{2} r^{2} \mathrm{~d} r \leq 0
$$

By using the fact that $\alpha R^{2} P \geq 0$, we obtain that the two integrals in the previous equation are equal to 0 . Therefore $c^{-}$is constant in space and in time. Using the boundary condition at $r=1$ leads to $c^{-}=0$, that is $C_{P_{\max }} \leq C$. The proof of the second inequality $C \leq C_{P_{\min }}$ is similar.

Corollary 8.4. The concentration $C$ is non-negative and increasing (i.e $\partial_{r} C \geq 0$ ).

Proof. The non-negativity of $C$ is a direct consequence of the maximum principle. Then, by integrating equation (6e) between 0 and $r$ and using the facts that $C$ and $P$ are non-negative we obtain $\partial_{r} C \geq 0$.

Corollary 8.5. The cell density satisfies $\partial_{r} P \geq 0$;

Proof. By differentiating in space the equation (6c) and by using the results of the previous propositions and the assumptions on $\gamma_{0}$ and $\gamma_{1}$ we obtain the following differential inequality on $\partial_{r} P$ :

$$
\partial_{t}\left(\partial_{r} P\right)+v \partial_{r}\left(\partial_{r} P\right) \geq\left(-\partial_{r} v+\gamma_{0}(C)(1-2 P)-\gamma_{1}(C)\right) \partial_{r} P
$$

Then using the characteristic curves defined by $v$ and the assumption that $\partial_{r} P^{0} \geq 0$ lead to the result. 
Proposition 8.6 (Estimate for the radius). Denote by $\beta=\gamma_{1}\left(C_{0}\right)-\gamma_{0}\left(C_{0}\right)$. The spheroid radius satisfies the following inequality:

$$
R(t) \leq R^{0}\left(1+\gamma_{0}\left(C_{0}\right) P^{0}(1) \frac{\left(1-e^{-\beta t}\right)}{\beta}\right)^{1 / 3} .
$$

Therefore, when $\gamma_{0}\left(C_{0}\right)<\gamma_{1}\left(C_{0}\right)$ the radius is bounded. Moreover, if the following assumptions hold:

- there is no proliferation gradient in the initial tumor (i.e the function $P^{0}$ is constant),

- the functions $\gamma_{0}(C)$ and $\gamma_{1}(C)$ are constant (i.e $\gamma_{0}(C)=\gamma_{0}\left(C_{0}\right)$ and $\gamma_{1}(C)=\gamma_{1}\left(C_{0}\right)$ ),

then the above inequality is an equality instead. In such case, for non quiescent tumor $\left(P^{0}>0\right)$, saturation is achieved if and only if $\beta>0$ (and the volume is linearly increasing in time if $\beta=0)$.

Proof. Thanks to Corollary (8.4) and Corollary 8.5, we obtain a uniform upper bound for $\gamma_{0}(C) P$ by using $\gamma_{0}\left(C_{0}\right)$ and the formula (26). Then we use this upper bound in equation (7) (page 6) to obtain an upper bound for $R^{\prime}(t)$. We conclude by applying Gronwall's inequality to obtain the above estimate for the radius.

The proof of the equality in the absence of proliferation gradient is twofold. First, the initial condition for $P$ and the assumption that $\gamma_{0}$ and $\gamma_{1}$ are constant implies there is no proliferation gradient for any time (thanks to equation (6d), the velocity vanishes in the absence of a proliferation gradient so that the equation for $P$ reduces to an ODE). Then, we observe that all the inequalities in the proof for the upper bound for $R$ become equalities in such a case.

Proposition 8.7. For any $t \geq 0$ and $r$ in $[0,1]$ the velocity satisfies:

$$
\text { - }(1 / 4)^{4 / 3}\left[\gamma_{0}(C(t, \cdot)) P(t, \cdot)\right]_{r=0}^{r=1} \leq g(r)\left[\gamma_{0}(C(t, \cdot)) P(t, \cdot)\right]_{r=0}^{r=1} \leq v(t, r) \leq 0,
$$

where $[f(\cdot)]_{r=0}^{r=1}=f(1)-f(0)$ for any function $f$ and $g$ is defined by

$$
g(r)=-\frac{r\left(1-r^{3}\right)}{3} .
$$

Proof. We start by rewriting equation $6 \mathrm{~d}$ ) by the following:

$$
v(t, r)=\frac{1}{r^{2}}\left[\left(1-r^{3}\right) \Gamma_{P}(t, r)+r^{3}\left(\Gamma_{P}(t, r)-\Gamma_{P}(t, 1)\right)\right] .
$$

Therefore $r^{2} v(t, r)$ is a convex combination of $\Gamma_{P}(t, r)$ and $\left(\Gamma_{P}(t, r)-\Gamma_{P}(t, 1)\right)$. From the assumptions, Proposition 8.1. and Corollary 8.5 we obtain that $r \longmapsto \gamma_{0}(C(t, \cdot)) P(t, \cdot)$ is positive and increasing. This leads to the following bounds for $\Gamma_{P}(t, r)$ and $\left(\Gamma_{P}(t, r)-\Gamma_{P}(t, 1)\right)$ :

$$
\begin{aligned}
\frac{r^{3}}{3}\left(\gamma_{0}(C) P\right)(t, 0) & \leq \Gamma_{P}(t, r) \leq \frac{r^{3}}{3}\left(\gamma_{0}(C) P\right)(t, r), \\
-\frac{1-r^{3}}{3}\left(\gamma_{0}(C) P\right)(t, 1) \leq \Gamma_{P}(t, r)-\Gamma_{P}(t, 1) & \leq-\frac{1-r^{3}}{3}\left(\gamma_{0}(C) P\right)(t, r) .
\end{aligned}
$$

Multiplying the first inequality by $\left(1-r^{3}\right) \geq 0$ and the second inequality by $r^{3} \geq 0$ and summing the two resulting inequalities lead to

$$
-\frac{r\left(1-r^{3}\right)}{3}\left[\gamma_{0}(C(t, \cdot)) P(t, \cdot)\right]_{r=0}^{r=1} \leq v(t, r) \leq 0 .
$$


Then by studying the function $g$ we prove that the minimum of $g$ is reached at $r_{*}=(1 / 4)^{\frac{1}{3}}$ and we have $g\left(r_{*}\right)=-(1 / 4)^{\frac{4}{3}}$.

Remark 8.8. In Proposition 8.7, the bound using the functiong is optimal. For every $r^{\prime} \in(0,1)$, if the function $\gamma_{0}(C(t, \cdot)) P(t, \cdot)$ is the characteristic function of the interval $\left[r^{\prime}, 1\right]$, then the velocity satisfies $v\left(t, r^{\prime}\right)=g\left(r^{\prime}\right)$. See Fig 9 for an example when $r^{\prime}=r_{*}$ is the argument of the minima of function $g$, as defined in the proof of Proposition 8.7.
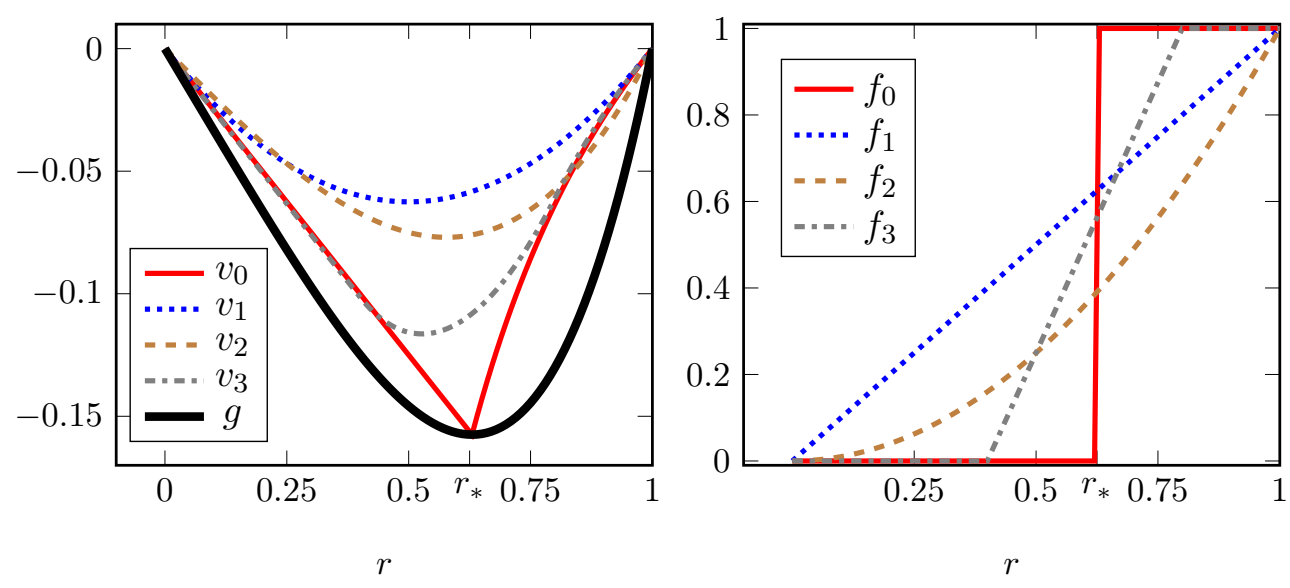

Figure 9: Plot of several velocity fields $v_{i}$ and of $g$ defined in Proposition 8.7 (left) for different functions $f_{i}=\gamma_{0}(C) P$ (right).

\subsection{Numerical simulation}

Here we give the outline of the numerical method used for the numerical simulation of the model.

For the spatial discretization, we use a finite difference method with $J=101$ points. We denote by

$$
\delta r=\frac{1}{J-1}
$$

the step size and by

$$
r_{j}=(j-1) \delta r, \quad \text { for } j=1, \cdots, J .
$$

For the time discretization we use a constant time step provided by the CFL condition given by Proposition 8.7 .

$$
\delta t=\frac{4^{4 / 3}}{\sup _{C \in[0,1]} \gamma_{0}(C)} \delta r
$$

and we denote by

$$
t^{n}=n \delta t
$$

In the following, we denote by $R^{n}, \Gamma_{j}^{n}, P_{j}^{n}, v_{j}^{n}$, respectively $C_{j}^{n}$ the approximation of $R\left(t^{n}\right)$, $\Gamma\left(r_{j}, t^{n}\right), P\left(r_{j}, t^{n}\right), v\left(r_{j}, t^{n}\right)$, respectively $C\left(r_{j}, t^{n}\right)$. 
For updating the radius and computing the velocity $v$, one needs to compute the integral $\Gamma_{j}^{n}$. For that purpose, we use the following formula:

$$
\Gamma_{j}^{n}=\sum_{k=1}^{j-1} \frac{\gamma_{0}\left(C_{j+1}^{n}\right) P_{j+1}^{n}+\gamma_{0}\left(C_{j}^{n}\right) P_{j}^{n}}{2} \frac{r_{k+1}^{3}-r_{k}^{3}}{3}
$$

This formula is similar to the middle-point formula applied to $\gamma_{0}(C) P$, where the middle-point is approximated by

$$
\frac{\gamma_{0}\left(C_{j+1}^{n}\right) P_{j+1}^{n}+\gamma_{0}\left(C_{j}^{n}\right) P_{j}^{n}}{2} .
$$

This formula is of order 2 but we do not use this property. The main advantage of this formula is to ensure the numerical velocity $v_{j}^{j}$ to satisfy the discrete version of Proposition 8.7 (the proof is similar to the continuous version, replacing integrals by sums). Moreover, the above formula is exact for $\gamma_{0}(C) P$ constant in space. By using $\Gamma_{j}^{n}$, we update the radius by explicit Euler method.

For computing the concentration $C_{j}^{n}$, we simply use the matrix defined from finite differences approximation of the 3D radial Laplacian operator and we solve the linear system with boundary conditions by using the LU method.

For updating the proliferation density $P_{j}^{n}$, we split the equation (6c) in 2 parts, the advection part and the reaction part, by using Lie splitting. For the advection part, we use the upwind scheme and denote by $P_{j}^{n *}$ the advected density. For the reaction part, we use an semi-implicit Euler method for stability, that is

$$
\frac{P_{j}^{n+1}-P_{j}^{n *}}{\delta t}=\gamma_{0}\left(C_{j}^{n *}\right) P_{j}^{n+1}\left(1-P_{j}^{n+1}\right)-\gamma_{1}\left(C_{j}^{n *}\right) P_{j}^{n+1}
$$

where $C_{j}^{n *}$ is computed by solving the equation with $R^{n+1}$ and $P_{j}^{n *}$.

\subsection{Choice for the function $\gamma_{1}$}

The function $\gamma_{1}(C)$ denotes the rate at which proliferating cells enter the quiescent state. This is the main parameter of the model since it drives the density of proliferating cells. The first idea is to consider a regularization of the unit step. For example, we can define $\gamma_{1}(C)$ by using the following formula:

$$
\tilde{\gamma_{1}}(C)=\gamma_{1}^{\min }+\left(\gamma_{1}^{\max }-\gamma_{1}^{\min }\right) \frac{1-\tanh \left(K\left(C-C_{h y p}\right)\right)}{2},
$$

where $\gamma_{1}^{\min }$ and $\gamma_{1}^{\max }$ stand for the infemum and the supremum of $\tilde{\gamma}_{1}$ (these values are not reached), $C_{\text {hyp }}$ is a hypoxia threshold, and $K$ is a parameter which controls the stiffness of $\tilde{\gamma_{1}}(C)$.

Numerical simulations were computed with several functions $\tilde{\gamma}_{1}$ defined by $(33)$ and are presented in Fig. 10. For these simulations, we used the same time span as the one in the data.

From these simulations, we can observe that:

1. in order to model a proliferating cell density which is flat near spheroid surface, as one can observe in the experimental data for the CAPAN-2 control experiment (see Figure 6), it seems necessary to use a function which is flat for concentrations near the maximum value; 

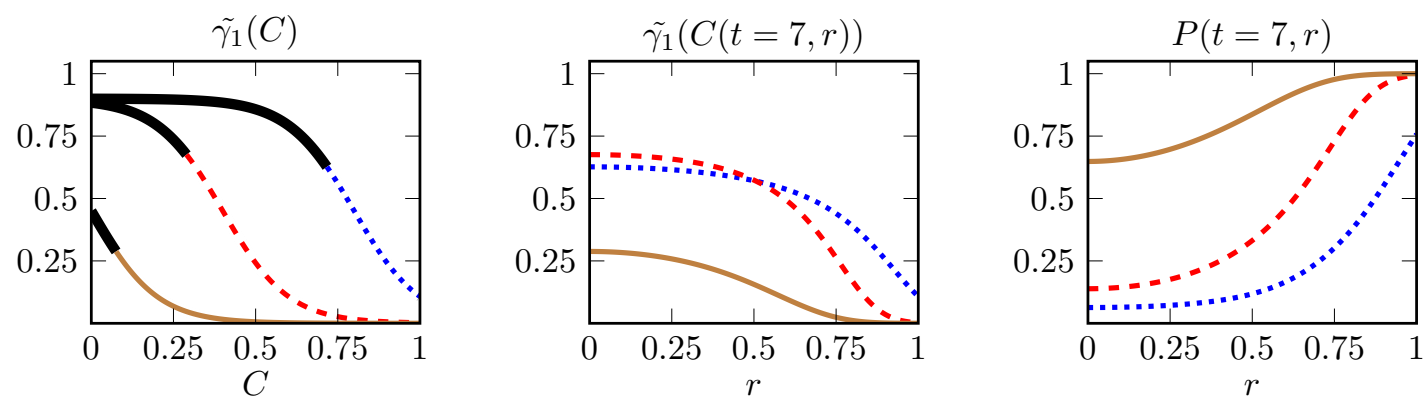

$$
\cdots C_{\text {hyp }}=0.8-\cdots C_{\text {hyp }}=0.4-C_{\text {hyp }}=0
$$

Figure 10: Plots of $\tilde{\gamma}_{1}(C)$ and $P$ for a constant $\gamma_{0}=0.4, \alpha=2, \gamma_{1}=0.9, K=5,3$ different values for $C_{\text {hyp }}$, and with initial conditions $R(t=0)=R^{0}=1.5$ and $P(t=0, r)=P^{0}(r)=1$. We plot in thick black the values of $\tilde{\gamma}_{1}(C)$ which are never reached during the simulation.

2. the minimum value of the switch rate must be positive in order to model a proliferating cells density that is not equal to 1 at the surface of the spheroid, as one can observe in the data. This property is a consequence of the formula (26) given in Section 8.1.

3. the left-hand side of the curve of the function $\tilde{\gamma}_{1}$ (for $C \simeq 0$ ) is never reached (see the thick black lines on the left of Fig. 10). Therefore, it seems that the qualitative behavior of the proliferation gradient observed in the data can be reproduced by using only the right-hand side of a sigmoid function.

Based on these observations, we decided to simplify the expression of switch rate and we chose the general expression (11) which has only 3 parameters. An other advantage of this formula is that the parameter $\gamma_{1, C_{0}}$ can be directly estimated from the experimental data and the equation satisfied by the density of proliferating cells at spheroid surface 26 . After trying several values of the parameter $m$ in (11) and comparing between the simulations and the data, we decided to fix it to 3 , so that the function $\gamma_{1}$ given by (11) has only 2 parameters to be estimated.

\subsection{Sensitivity of the model}

In this section, we briefly study the sensitivity of the model with respect to the parameters $m, a$, and $\alpha$. We observe that the range for the parameters $a$ and $\alpha$ can vary markedly and the optimal values for $a$ and $\alpha$ are highly dependent on $m$. Our intuition is that the qualitative behavior of the proliferation gradient is driven by the qualitative behavior of the function $(t, r) \longmapsto \gamma_{1}(C(t, r))$ and that such behavior can be approximated by a wide subspace of the parameter space for $m$, $a$, and $\alpha$.

We also observe that the parameters $\gamma_{0}$ and $\gamma_{1, C_{0}}$ do not change significantly while $m$ vary. This is because these two parameters are almost driven explicitly by the data: $\gamma_{0}$ is mainly driven by the dynamics of the radius and the dynamics of the total amount of proliferating cells (9) while $\gamma_{1, C_{0}}$ is mainly driven by the proliferating cell density at spheroid surface (26). However, the parameter $\gamma_{1, C_{0}}$ seems to vary with $m$ for the HCT-116 experiments, but in that case we suspect this is due to the fact that there is only one time point to fit. 


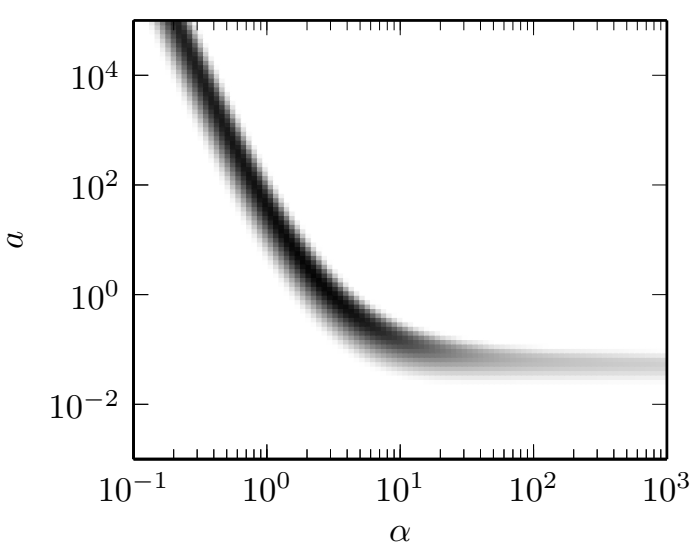

$\min (\varepsilon, 1.5 \min \varepsilon)$

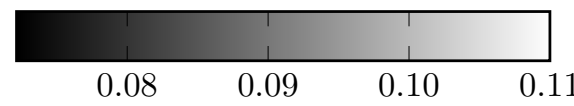

(a) CAPAN-2 control.

$\gamma_{0}^{0}=3.68 \cdot 10^{-1}$ and $\gamma_{1, C_{0}}^{0}=1.44 \cdot 10^{-1}$

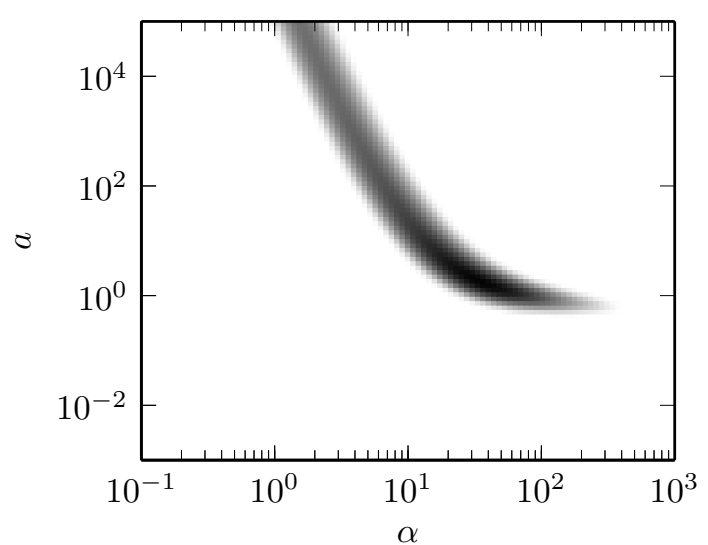

$\min (\varepsilon, 1.5 \min \varepsilon)$

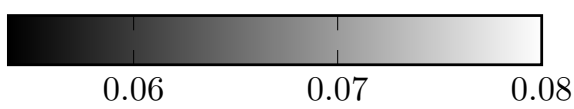

(b) CAPAN-2 quiescent.

$\gamma_{0}^{0}=6.18 \cdot 10^{-1}$ and $\gamma_{1, C_{0}}^{0}=8.23 \cdot 10^{-1}$

Figure 11: Plot of the discrepancy values obtained during the scanning of the parameter space for $\alpha$ and $a$ during the initialization step described in Section 3.3 . Discrepancy is computed by using the initial estimated values $\gamma_{0}^{0}$ and $\gamma_{1, C_{0}}^{0}$ defined in Section 3.3 with $m=3$. For each plot, the values above 1.5 min $\varepsilon$ have been truncated to improve clarity.

\begin{tabular}{|c|c|c|c|c|c|c|}
\hline & \multicolumn{5}{|c|}{ parameters } & \multirow{2}{*}{$\begin{array}{c}\text { discrepancy } \\
\varepsilon \\
\end{array}$} \\
\hline & $m$ & $\gamma_{0}$ & $\gamma_{1, C_{0}}$ & $a$ & $\alpha$ & \\
\hline \multirow{3}{*}{ CAPAN-2 control } & 1 & $3.58 \mathrm{e}-01$ & $1.18 \mathrm{e}-01$ & $1.92 \mathrm{e}+01$ & $1.75 \mathrm{e}-01$ & $7.97 \mathrm{e}-02$ \\
\hline & 2 & $3.58 \mathrm{e}-01$ & $1.33 \mathrm{e}-01$ & $7.57 \mathrm{e}+00$ & $9.66 \mathrm{e}-01$ & $7.44 \mathrm{e}-02$ \\
\hline & 3 & $3.58 \mathrm{e}-01$ & $1.37 \mathrm{e}-01$ & $2.07 \mathrm{e}+00$ & $2.40 \mathrm{e}+00$ & $7.18 \mathrm{e}-02$ \\
\hline \multirow{3}{*}{ CAPAN-2 quiescent } & 1 & $6.90 \mathrm{e}-01$ & $8.21 \mathrm{e}-01$ & $5.77 \mathrm{e}+00$ & $5.43 \mathrm{e}+00$ & $5.59 \mathrm{e}-02$ \\
\hline & 2 & $6.78 \mathrm{e}-01$ & $8.27 \mathrm{e}-01$ & $1.90 \mathrm{e}+00$ & $2.96 \mathrm{e}+01$ & $5.27 \mathrm{e}-02$ \\
\hline & 3 & $6.74 \mathrm{e}-01$ & $8.51 \mathrm{e}-01$ & $1.91 \mathrm{e}+00$ & $3.85 \mathrm{e}+01$ & $5.20 \mathrm{e}-02$ \\
\hline \multirow{3}{*}{ HCT-116 21\% O } & 1 & $6.29 \mathrm{e}-01$ & $9.28 \mathrm{e}-07$ & $2.85 \mathrm{e}+01$ & $6.06 \mathrm{e}-05$ & $3.07 \mathrm{e}-02$ \\
\hline & 2 & $6.38 \mathrm{e}-01$ & $3.73 \mathrm{e}-02$ & $1.36 \mathrm{e}+00$ & $9.89 \mathrm{e}-04$ & $2.80 \mathrm{e}-02$ \\
\hline & 3 & $6.39 \mathrm{e}-01$ & $4.75 \mathrm{e}-02$ & $1.03 \mathrm{e}+00$ & $1.72 \mathrm{e}-03$ & $2.70 \mathrm{e}-02$ \\
\hline \multirow{3}{*}{ HCT-116 5\% O ${ }_{2}$} & 1 & $3.20 \mathrm{e}-01$ & $2.56 \mathrm{e}-02$ & $9.14 \mathrm{e}+01$ & $5.17 \mathrm{e}-05$ & $4.26 \mathrm{e}-02$ \\
\hline & 2 & $3.19 \mathrm{e}-01$ & $5.37 \mathrm{e}-02$ & $1.30 \mathrm{e}+00$ & $1.41 \mathrm{e}-03$ & $3.64 \mathrm{e}-02$ \\
\hline & 3 & $3.19 \mathrm{e}-01$ & $5.95 \mathrm{e}-02$ & $8.06 \mathrm{e}-01$ & $2.75 \mathrm{e}-03$ & $3.36 \mathrm{e}-02$ \\
\hline
\end{tabular}

Table 2: Sensibility of the parameters estimation with respect to different values of the parameter $m$. The calibrations were performed by using algorithm of Section 3 on the different datasets with fixed value for $m$. The discrepancy $\varepsilon$ is defined in Definition 3.7 . 